\title{
Improvement of accuracy in the wave-function-matching method for transport calculations
}

\author{
Shigeru Tsukamoto, ${ }^{1, *}$ Tomoya Ono, ${ }^{2, \dagger}$ and Stefan Blügel ${ }^{1}$ \\ ${ }^{1}$ Peter Grünberg Institut \& Institute for Advanced Simulation, Forschungszentrum Jülich and JARA, D-52425 Jülich, Germany \\ ${ }^{2}$ Center for Computational Sciences, University of Tsukuba, Tsukuba, Ibaraki 305-8577, Japan
}

(Received 27 October 2017; revised manuscript received 29 January 2018; published 28 March 2018)

\begin{abstract}
The wave-function-matching (WFM) technique for first-principles transport-property calculations was bettered by Sørensen et al. so as to exclude rapidly decreasing evanescent waves [Sørensen et al., Phys. Rev. B 77, 155301 (2008)]. In their method, the translational invariance of the transmission probability is not preserved when moving the matching planes between electrode and transition regions, and the sum of transmission and reflection probabilities does not agree with the number of transport channels in the transition region. The lack of the translational invariance is caused by the overlap of the layers between the electrode and transition regions. We reformulate the WFM method by removing the layer overlap, and the translational invariance of the transmission probability becomes preserved. On the other hand, the error in the sum of transmission and reflection probabilities is attributed to using pseudoinverses that is accompanied by the exclusion of rapidly decreasing evanescent waves. We introduce a formulation to calculate the transmission/reflection probability without the pseudoinverses, resulting in that the sum of the transmission and reflection probabilities exactly agrees with the number of channels, and the accuracy is largely improved. In addition, we prove that the accuracy in the transmission probability obtained by our WFM technique is comparable to that obtained by a nonequilibrium Green's function method. Furthermore, we carry out electron transport calculations on two-dimensional graphene sheets embedded with B-N line defects sandwiched between a pair of semi-infinite graphene electrodes and find the dependence of the electron transmission on the transverse momentum perpendicular to the transport direction.
\end{abstract}

DOI: 10.1103/PhysRevB.97.115450

\section{INTRODUCTION}

The study of electron transport in nanoscale systems is becoming important as the miniaturization of electronic devices proceeds because they are expected to exhibit considerably different transport properties from those of classical conductors. Owing to the complexity of the problem, such studies are strongly dependent on the existence of reliable numerical treatments. A number of numerical methods for calculating the electron-transport properties of nanoscale systems have been proposed so far, and some of them are combined with first-principles calculations. The methods used currently in the first-principles calculations are roughly categorized into two approaches. One approach uses the nonequilibrium Green's function [1-4]. The relation between the conductance and Green's function has been derived within the nonequilibrium Keldysh formalism [5], and the charge density in the equilibrium regime of energy is easily computed with the energy of a nonreal number. The other approach is the so-called wave-function-matching (WFM) method, which computes the scattering wave functions (SWFs) providing a direct realspace picture of the scattering process [6-15]. Both methods have the computational models in which a transition region composed of objective nanostructures is sandwiched between semi-infinitely continuing electrodes. In the Green's function formalism, the self-energy terms of the electrodes reflect the

\footnotetext{
*s.tsukamoto@fz-juelich.de

†ono@ccs.tsukuba.ac.jp
}

effect from the semi-infinite electrodes and the perturbed Green's functions of the transition region are computed using the self-energy terms. The conductance of a system is obtained from the Fisher-Lee formula [16] by using the perturbed Green's functions and the self-energy terms of electrodes. On the other hand, in the WFM method, the conductance is expressed as a quantum mechanical scattering problem. The generalized Bloch waves of the electrodes are used to include the contribution of the semi-infinite electrodes in the WFM formula. The conductance and total transmission probability are related to the transmission coefficients and the group velocities of the Bloch waves by the Landauer formula [17]. It has been proved that the methods are mathematically equivalent [14] and the conductances obtained by them should be identical.

Several WFM methods have been proposed so far, and their formulations are slightly different. The computations used to find the generalized Bloch waves of the electrodes are generally time consuming and numerically unstable because of treating the extremely large and small eigenvalues corresponding to the Bloch factors [11]. On the basis of the physical observation that only propagating and slowly decaying evanescent waves of the electrodes contribute to the transmission of electrons, rapidly decaying evanescent waves can be excluded by introducing a cutoff parameter for the Bloch factor $\lambda_{\min }\left(\lambda_{\min } \ll 1\right)$ in some WFM methods $[13,18-20]$. There have been several discussions on the translational invariance of the transport properties with respect to moving the matching planes between electrode and transition regions for the WFM formalism using the cutoff parameter $\lambda_{\min }$. Krstić et al. state that Ando's formulation for the scattering process lacks translational invariance [6,21], and 


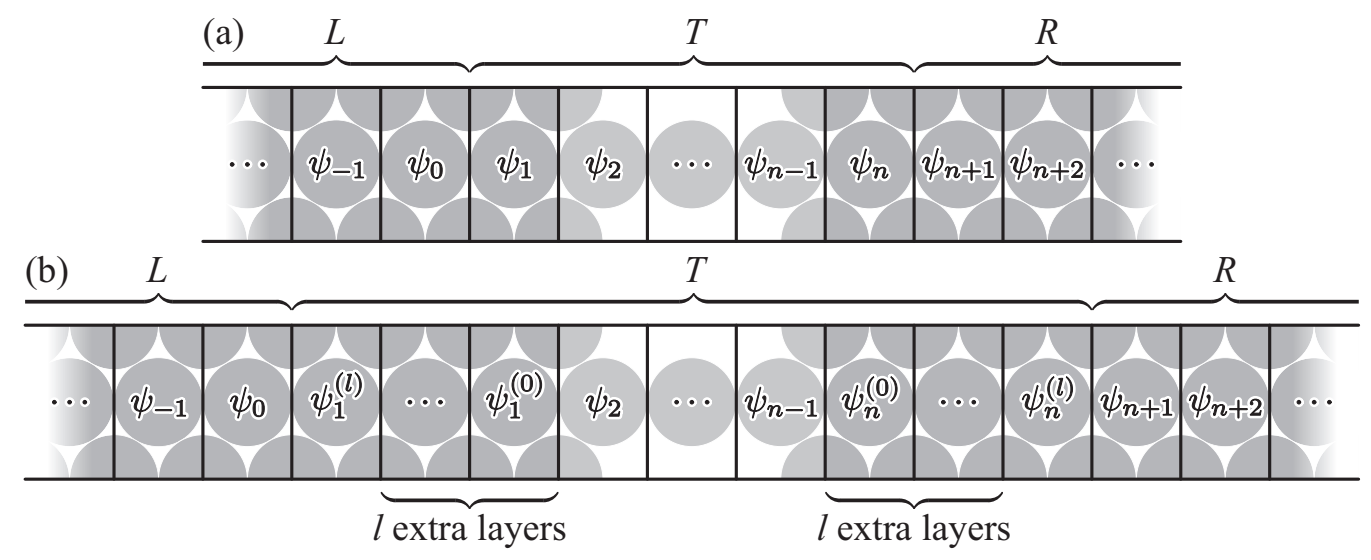

FIG. 1. Schematic illustration of computational model for WFM formalism. The transition region between the two semi-infinite electrodes represents the objective nanostructures. (a) Model without extra layers in the transition region and (b) model with $l$ extra layers.

later, Khomyakov et al. prove that the translational invariance is retained when the matching planes are moved [13]. Sørensen et al. report that the accuracy of transmission probability is degraded when the rapidly varying evanescent waves are excluded [18]. In addition, the inconsistency in the numerical errors between the transmission and reflection probabilities indicates that the sum of these probabilities does not agree with the number of transport channels. They also proposed a method that extends the transition region by attaching extra layers to both ends so as to converge the transmission/reflection probability to a certain value. However, from the viewpoint of the penetration of quantum particles as well as the proof by Khomyakov et al., the improvement by Sørensen et al. contradicts the fact that the transmission probability does not change when the extra layers are added. Therefore, the problems concerning the translational invariance and the sum of the transmission and reflection probabilities are important issues to be resolved for the present WFM methods.

In this paper, we reformulate the WFM formalism because most of the WFM methods that have been introduced so far include all the propagating and evanescent waves. Then, we explore the origin of the deterioration of the translational invariance presented by Sørensen et al. It is revealed that the translational invariance deteriorates due to the overlap of the layers between the electrode and transition regions when excluding rapidly decreasing evanescent waves. Moreover, the error in the transmission probability with respect to the number of extra layers demonstrated in Ref. [18] is not due to the WFM methods but rather due to the usage of pseudoinverses in the calculation of transmission coefficients. For a more accurate calculation of transport properties, we propose a method that can compute the transmission and reflection probabilities without inclusion of the rapidly varying evanescent waves or the extension of the transition region. We find that, in our WFM formalism, the translational invariance is nicely preserved, and the sum of the probabilities exactly agrees with the number of transport channels. We also find that the number of iterations in solving a continued-fraction equation for the self-energy terms of the electrodes is closely related to the number of the extra layers attached to the transition region. We demonstrate that the numerical accuracy of our WFM formalism is comparable to that of the nonequilibrium Green's function method even when the rapidly varying evanescent waves are not explicitly computed and the transition region is not extended. The algorithm of the present method to calculate the generalized Bloch states, scattering wave functions, and transmission probability based on the discussion in Sec. II is described in Fig. S1 in the Supplemental Material [22].

In addition to the improvement of the accuracy in the WFM calculations, we perform practical electron transport calculations of two-dimensional graphene sheets embedded with B-N line defects, which are connected to a pair of semi-infinite graphene electrodes. Graphene sheets are known to have only a characteristic band structure at around the Fermi energy $E_{\mathrm{F}}$, the so-called Dirac cone. This means that the electrons passing through the junctions are provided only from the Dirac cone and have a limited range of momenta. Through the transport calculations, we present the case that the defect states not matching with the incident-wave modes do not directly contribute to the electron transport, e.g., through resonant transport, but they have indirect influence on the transport properties via hybridization of the Dirac cone and the defect states.

\section{FORMALISM}

\section{A. Generalized Bloch waves in electrodes}

Let us introduce the WFM formalism for calculating the solution of the Kohn-Sham equation [31,48] of a system with a transition region sandwiched between semi-infinitely continuing crystalline electrodes, as shown in Fig. 1(a). We assume that in the layers at both ends of the transition region, which are indexed by 1 and $n$ in Fig. 1(a), physical quantities such as charge density and potential are converged to those in the corresponding electrodes. The solution we wish to calculate is the SWFs specified by particular incident Bloch waves coming from deep inside the left electrode. The SWFs for the Bloch waves coming from the right electrode can be obtained in a similar manner. Since the Kohn-Sham effective potential in a crystalline electrode is periodic, the wave functions $\phi_{(j)}$ in the $j$ th unit cell of the electrode satisfy the generalized Bloch condition,

$$
\phi_{(j)}=\lambda^{j-i} \phi_{(i)},
$$


where $i$ and $j$ are the indices of the unit cell of the crystal. Making use of the generalized Bloch condition, the wave functions in the left electrode $\phi_{(i)}$ are obtained by solving the following quadratic eigenvalue problem for the given energy $E[6]$;

$$
\overline{\mathbf{H}}_{L, L}^{\dagger} \phi_{(i)}+\lambda \overline{\mathbf{H}}_{L} \phi_{(i)}+\lambda^{2} \overline{\mathbf{H}}_{L, L} \phi_{(i)}=0 .
$$

Here, $\overline{\mathbf{H}}_{L}$ is the $m_{L} \times m_{L}$ full rank matrix for the Hamiltonian of the periodic unit cell with $m_{L}$ being the number of real-space grids or bases in a unit cell and $\overline{\mathbf{H}}_{L} \equiv E \mathbf{S}_{L}-\mathbf{H}_{L}$ with $\mathbf{S}_{L}$ being the overlap matrix of the bases. If one uses a real-space grid method and norm-conserving pseudopotentials [23], $\mathbf{S}_{L}$ is an identity matrix. $\overline{\mathbf{H}}_{L, L}$ is the $m_{L} \times m_{L}$ off-diagonal block matrix of the infinite Hamiltonian of the electrode representing the coupling between two neighboring unit cells. Because $\overline{\mathbf{H}}_{L, L}$ is not a full rank matrix in some cases, the rank of $\overline{\mathbf{H}}_{L, L}$ is represented by the other variable $m_{L L}$. In the case of $m_{L}>$ $m_{L L}$, we assume that $\overline{\mathbf{H}}_{L L}$ is a zero matrix except that the lower left $m_{L L} \times m_{L L}$ block-matrix element is $\overline{\mathbf{h}}_{L}$,

$$
\overline{\mathbf{H}}_{L, L}=\left(\begin{array}{ccc}
0 & \ldots & 0 \\
\vdots & \ddots & \vdots \\
\overline{\mathbf{h}}^{L} & \ldots & 0
\end{array}\right) .
$$

This assumption does not affect generality because $\overline{\mathbf{H}}_{L L}$ can be described using Eq. (3) by the unitary transformation [24,25]. The solution of Eq. (2) is obtained by solving the following eigenvalue problem:

$$
\left(\begin{array}{cc}
\overline{\mathbf{H}_{L}} & \overline{\mathbf{H}}_{L, L}^{\dagger} \\
\mathbf{I} & \mathbf{0}
\end{array}\right)\left(\begin{array}{c}
\phi_{(i)} \\
\phi_{(i-1)}
\end{array}\right)=\lambda\left(\begin{array}{cc}
-\overline{\mathbf{H}}_{L, L} & \mathbf{0} \\
\mathbf{0} & \mathbf{I}
\end{array}\right)\left(\begin{array}{c}
\phi_{(i)} \\
\phi_{(i-1)}
\end{array}\right) .
$$

Ando proposed a method of solving Eq. (4) as a standard eigenvalue problem, computational cost of which is $O\left(m_{L}^{3}\right)$ [6].

In 2008, Sørensen et al. reported a procedure that obtains selected interior eigenpairs of large-scale general complex eigenproblems, $\lambda_{\min }<|\lambda|<1 / \lambda_{\min }$, by using an iterative Krylov subspace technique, where $\lambda_{\min }$ is the cutoff parameter of the Bloch factor [4]. Laux proposed a practical approach which resolves only eigenpairs within a contour defined in the complex $\lambda$ plane [26]. Later, this approach of utilizing contour integrations was applied to the real-space grid schemes with some improvements in the Sakurai-Sugiura method [20,27,28]. Although they do not obtain all eigenpairs required to treat a semi-infinite system, these approaches are valid within the assumption that only the propagating and slowly decreasing evanescent waves contribute to the transport properties $[13,18]$. The alternative method to compute generalized Bloch waves is introduced in Appendix A.

The generalized Bloch waves are evenly divided into two groups, and the number of waves in a group is $m_{L L}$. The eigenpairs $\lambda_{\kappa}^{-}$and $\phi_{(i), \kappa}^{-}\left(\lambda_{\kappa}^{+}\right.$and $\left.\phi_{(i), \kappa}^{+}\right)$represent the group of the left (right) decreasing evanescent waves $\left|\lambda_{\kappa}\right|>0\left(\left|\lambda_{\kappa}\right|<0\right)$ and left (right) propagating waves $\left|\lambda_{\kappa}\right|=1$, where $\kappa$ is the index of eigenpairs. We also introduce the Bloch matrix, which relates the generalized Bloch waves with those in the neighboring cells,

$$
\mathbf{B}_{L}^{ \pm}=\boldsymbol{\Phi}_{L(i+1)}^{ \pm}\left(\boldsymbol{\Phi}_{L(i)}^{ \pm}\right)^{-1}=\boldsymbol{\Phi}_{L(i)}^{ \pm} \boldsymbol{\Lambda}_{L}^{ \pm}\left(\boldsymbol{\Phi}_{L(i)}^{ \pm}\right)^{-1},
$$

where $\boldsymbol{\Lambda}_{L}^{ \pm}=\operatorname{diag}\left[\lambda_{1}^{ \pm}, \ldots, \lambda_{m_{L L}}^{ \pm}\right]$and $\boldsymbol{\Phi}_{L(i)}^{ \pm}=\left[\phi_{L(i), 1}^{ \pm}, \ldots\right.$, $\phi_{L(i), m_{L L}}^{ \pm}$. Equation (5) leads to the relation,

$$
\boldsymbol{\Phi}_{L(j)}^{ \pm}=\left(\mathbf{B}_{L}^{ \pm}\right)^{j-i} \boldsymbol{\Phi}_{L(i)}^{ \pm}=\boldsymbol{\Phi}_{L(i)}^{ \pm}\left(\boldsymbol{\Lambda}_{L}^{ \pm}\right)^{j-i} .
$$

Hereafter, the index $(i)$ for the unit cell is left out because it only affects the trivial Bloch factor in Eq. (6). For the right electrode, the same quantities are defined with $L \rightarrow R$.

\section{B. Expression using transmission and reflection coefficients}

In the WFM formalism, the SWFs in the right electrode region $\psi_{n+1, k}$ are expressed as a linear combination of generalized Bloch waves,

$$
\psi_{n+1, k}=\boldsymbol{\Phi}_{R}^{+} \boldsymbol{t}_{k},
$$

where $\boldsymbol{t}_{k}$ is an $m_{R}$-dimensional vector containing the transmission coefficients and $k$ is the index of the incident waves. The SWFs in the left electrode region $\psi_{0, k}$ are defined as

$$
\psi_{0, k}=\boldsymbol{\Phi}_{L}^{-} \boldsymbol{r}_{k}+\phi_{L, k}^{+}=\psi_{0, k}^{\mathrm{ref}}+\psi_{0, k}^{\mathrm{in}},
$$

where

$$
\begin{gathered}
\psi_{0, k}^{\mathrm{ref}}=\boldsymbol{\Phi}_{L}^{-} \boldsymbol{r}_{k}, \\
\psi_{0, k}^{\text {in }}=\phi_{L, k}^{+}
\end{gathered}
$$

with $\boldsymbol{r}_{k}$ being an $m_{L}$-dimensional vector containing the reflection coefficients. Here, $\psi_{0, k}^{\mathrm{ref}}$ and $\psi_{0, k}^{\text {in }}$ represent the reflection and incident waves, respectively. In addition, the transmission and reflection coefficients are given by

$$
\begin{gathered}
\boldsymbol{t}_{k}=\left(\boldsymbol{\Phi}_{R}^{+}\right)^{-1} \psi_{n+1, k}, \\
\boldsymbol{r}_{k}=\left(\boldsymbol{\Phi}_{L}^{-}\right)^{-1} \psi_{0, k}^{\mathrm{ref}} .
\end{gathered}
$$

\section{Exclusion of rapidly varying evanescent waves}

The coefficients for the extremely fast decaying evanescent waves are very small when the matching planes are set far from the scatterers. Sørensen et al. split $\boldsymbol{\Phi}^{ \pm}$into propagating and moderately decaying evanescent waves $\widetilde{\boldsymbol{\Phi}}^{ \pm}$and rapidly decaying evanescent waves $\stackrel{\circ}{\Phi}^{ \pm}[18]$

$$
\boldsymbol{\Phi}^{ \pm}=\left[\widetilde{\boldsymbol{\Phi}}^{ \pm}, \stackrel{\Phi}{\Phi}^{ \pm}\right] .
$$

The SWFs at the first layers of the right and left electrode regions are rewritten as

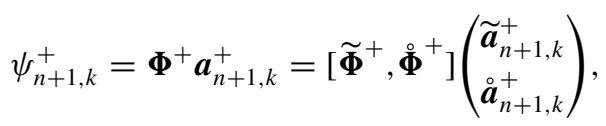

and

$$
\psi_{0, k}^{\mathrm{ref},-}=\boldsymbol{\Phi}^{-} \boldsymbol{a}_{0, k}^{\mathrm{ref},-}=\left[\widetilde{\boldsymbol{\Phi}}^{-}, \stackrel{\boldsymbol{\Phi}}{ }^{-}\right]\left(\begin{array}{c}
\widetilde{\boldsymbol{a}}_{0, k}^{\mathrm{ref},-} \\
\dot{\boldsymbol{a}}_{0, k}^{\mathrm{ref},-}
\end{array}\right),
$$

respectively, where $\boldsymbol{a}_{i, k}^{ \pm}=\left[\widetilde{\boldsymbol{a}}_{i, k}^{ \pm T}, \stackrel{\stackrel{a}{a}_{i, k}^{ \pm T}}{]^{T}}\right.$ are vectors that contain the expansion coefficients of the generalized Bloch waves.

It is computationally demanding and numerically unstable to obtain all eigenpairs of Eq. (4). To retain numerical accuracy 
TABLE I. Electrode and transition regions for the WFM methods proposed so far.

\begin{tabular}{lccc}
\hline \hline & Left electrode region & Transition region & Right electrode region \\
\hline Fujimoto \& Hirose [11] & $\ldots,-1,0$ & $0,1, \ldots, n, n+1$ & $n+1, n+2, \ldots$ \\
Khomyakov et al. $[13]$ & $\ldots,-1,0$ & $0,1, \ldots, n, n+1$ & $n+1, n+2, \ldots$ \\
Sørensen et al. $[18]$ & $\ldots, 0,1$ & $1,2, \ldots, n-1, n$ & $n, n+1, \ldots$ \\
Present work & $\ldots,-1,0$ & $1,2, \ldots, n-1, n$ & $n+1, n+2, \ldots$ \\
\hline \hline
\end{tabular}

without $\stackrel{\Phi}{\Phi}^{ \pm}$, Sørensen et al. proposed a method that inserts extra layers in the transition region, as shown in Fig. 1(b). Making use of the relation $\mathbf{B}^{ \pm}=\boldsymbol{\Phi}^{ \pm} \boldsymbol{\Lambda}^{ \pm}\left(\boldsymbol{\Phi}^{ \pm}\right)^{-1}$, the SWFs in the electrode regions are expressed as

$$
\begin{aligned}
& \psi_{n+1, k}^{+}=\left(\mathbf{B}_{R}^{+}\right)^{l} \psi_{n, k}^{(0),+}=\left(\widetilde{\boldsymbol{\Phi}}_{R}^{+}, \stackrel{\circ}{\boldsymbol{\Phi}}_{R}^{+}\right)\left(\begin{array}{c}
\left(\widetilde{\boldsymbol{\Lambda}}_{R}^{+}\right)^{l} \widetilde{\boldsymbol{a}}_{n, k}^{(0),+} \\
\left.\stackrel{\boldsymbol{\Lambda}}{R}^{+}\right)^{l} \stackrel{\circ}{\boldsymbol{a}}_{n, k}^{(0),+}
\end{array}\right), \\
& \psi_{0, k}^{\mathrm{ref},-}=\left(\mathbf{B}_{L}^{-}\right)^{-l} \psi_{1, k}^{\mathrm{ref},(0),-}
\end{aligned}
$$

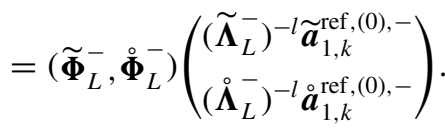

From Eqs. (11) and (12), we have

$$
\begin{aligned}
& \boldsymbol{t}_{k}=\left(\begin{array}{c}
\tilde{\boldsymbol{t}}_{k} \\
\mathfrak{\boldsymbol { t }}_{k}
\end{array}\right)=\left(\widetilde{\boldsymbol{\Phi}}_{R}^{+}, \stackrel{\circ}{\boldsymbol{\Phi}}_{R}^{+}\right)^{-1} \psi_{n+1, k}^{+}=\left(\begin{array}{c}
\left(\widetilde{\boldsymbol{\Lambda}}_{R}^{+}\right)^{l} \widetilde{\boldsymbol{a}}_{n, k}^{(0),+} \\
\left(\stackrel{\boldsymbol{\Lambda}}{R}_{R}^{+}\right)^{l} \stackrel{\boldsymbol{a}}{n, k}_{n,+}^{(0),+}
\end{array}\right), \\
& \boldsymbol{r}_{k}=\left(\begin{array}{c}
\widetilde{\boldsymbol{r}}_{k} \\
\boldsymbol{r}_{k}
\end{array}\right)=\left(\widetilde{\boldsymbol{\Phi}}_{L}^{-}, \stackrel{\circ}{\boldsymbol{\Phi}}_{L}^{-}\right)^{-1} \psi_{0, k}^{\mathrm{ref},-}=\left(\begin{array}{c}
\left(\tilde{\boldsymbol{\Lambda}}_{L}^{-}\right)^{-l} \widetilde{\boldsymbol{a}}_{1, k}^{\mathrm{ref},(0),-} \\
\left(\stackrel{\boldsymbol{\Lambda}}{L}^{-}\right)^{-l} \stackrel{\mathrm{a}}{1, k}_{\mathrm{ref},(0),-}
\end{array}\right) .
\end{aligned}
$$

The coefficients of the rapidly decaying evanescent waves vanish when a sufficient number of extra layers are inserted, because the Bloch factors of the rapidly decaying evanescent waves, $1 /\left|\lambda_{L}^{-}\right|\left(<\lambda_{\text {min }}\right)$ and $\left|\lambda_{R}^{+}\right|\left(<\lambda_{\text {min }}\right)$, are much smaller than 1 .

$$
\begin{gathered}
\left(\stackrel{\circ}{\Lambda}_{R}^{+}\right)^{l} \stackrel{\leftrightarrow}{\boldsymbol{a}}_{n, k}^{(0),+} \approx \mathbf{0}, \\
\left(\stackrel{\circ}{L}_{L}^{-}\right)^{-l} \stackrel{\mathrm{a}}{1, k}^{\mathrm{ref},(0),-} \approx \mathbf{0} .
\end{gathered}
$$

Consequently, the SWFs at the first layers of the electrode regions are rewritten as

$$
\begin{aligned}
& \psi_{n+1, k}=\psi_{n+1, k}^{+}=\left(\widetilde{\boldsymbol{\Phi}}_{R}^{+}, \stackrel{\boldsymbol{\Phi}}{R}_{R}^{+}\right)\left(\begin{array}{c}
\left(\widetilde{\boldsymbol{\Lambda}}_{R}^{+}\right)^{l} \widetilde{\boldsymbol{a}}_{n, k}^{(0),+} \\
\left(\stackrel{\boldsymbol{\Lambda}}{R}_{R}^{+}\right)^{l} \stackrel{\boldsymbol{a}}{n, k}_{n,+}^{(0),+}
\end{array}\right) \\
& \approx \widetilde{\boldsymbol{\Phi}}_{R}^{+}\left(\widetilde{\boldsymbol{\Lambda}}_{R}^{+}\right)^{l} \widetilde{\boldsymbol{a}}_{n, k}^{(0),+}, \\
& \psi_{0, k}=\psi_{0, k}^{\mathrm{ref}}+\psi_{0, k}^{\mathrm{in}} \text {, } \\
& \psi_{0, k}^{\mathrm{ref}}=\psi_{0, k}^{\mathrm{ref},-}=\left(\widetilde{\boldsymbol{\Phi}}_{L}^{-}, \stackrel{\AA}{\boldsymbol{\Phi}}_{L}^{-}\right)\left(\begin{array}{l}
\left(\tilde{\boldsymbol{\Lambda}}_{L}^{-}\right)^{-l} \widetilde{\boldsymbol{a}}_{1, k}^{\mathrm{ref},(0),-} \\
\left(\stackrel{\boldsymbol{\Lambda}}{L}_{L}^{-}\right)^{-l} \stackrel{\mathrm{a}}{1, k}^{\mathrm{ref},(0),-}
\end{array}\right) \\
& \approx \widetilde{\boldsymbol{\Phi}}_{L}^{-}\left(\tilde{\boldsymbol{\Lambda}}_{L}^{-}\right)^{-l} \widetilde{\boldsymbol{a}}_{1, k}^{\mathrm{ref},(0),-} \\
& \psi_{0, k}^{\text {in }}=\left(\lambda_{L, k}^{+}\right)^{-l} \phi_{L, k}^{+} \text {. }
\end{aligned}
$$

The SWFs at the second layers in the electrode regions are expressed in the same manner:

$$
\begin{aligned}
& \psi_{n+2, k}=\psi_{n+2, k}^{+}=\left(\widetilde{\boldsymbol{\Phi}}_{R}^{+} \widetilde{\boldsymbol{\Lambda}}_{R}^{+}, \stackrel{\circ}{\boldsymbol{\Phi}}_{R}^{+} \stackrel{\circ}{\boldsymbol{\Lambda}}_{R}^{+}\right)\left(\begin{array}{c}
\left(\widetilde{\boldsymbol{\Lambda}}_{R}^{+}\right)^{l} \widetilde{\boldsymbol{a}}_{n, k}^{(0),+} \\
\left(\stackrel{\boldsymbol{\Lambda}}{R}^{+}\right)^{l} \stackrel{\circ}{\boldsymbol{a}}_{n, k}^{(0),+}
\end{array}\right) \\
& \approx \widetilde{\boldsymbol{\Phi}}_{R}^{+}\left(\widetilde{\boldsymbol{\Lambda}}_{R}^{+}\right)^{l+1} \widetilde{\boldsymbol{a}}_{n, k}^{(0),+}, \\
& \psi_{-1, k}=\psi_{-1, k}^{\mathrm{ref}}+\psi_{-1, k}^{\text {in }} \text {, } \\
& \psi_{-1, k}^{\mathrm{ref}}=\psi \psi_{-1, k}^{\mathrm{ref},-}=\left(\widetilde{\boldsymbol{\Phi}}_{L}^{-}\left(\tilde{\boldsymbol{\Lambda}}_{L}^{-}\right)^{-1}, \stackrel{\circ}{\boldsymbol{\Phi}}_{L}^{-}\left(\stackrel{\boldsymbol{\Lambda}}{L}_{L}^{-}\right)^{-1}\right)\left(\begin{array}{l}
\left(\widetilde{\boldsymbol{\Lambda}}_{L}^{-}\right)^{-l} \widetilde{\boldsymbol{a}}_{1, k}^{\mathrm{ref},(0),-} \\
\left(\stackrel{\boldsymbol{\Lambda}}{L}_{L}^{-}\right)^{-l} \stackrel{\circ}{\boldsymbol{a}}_{1, k}^{\mathrm{ref},(0),-}
\end{array}\right) \\
& \approx \widetilde{\boldsymbol{\Phi}}_{L}^{-}\left(\widetilde{\boldsymbol{\Lambda}}_{L}^{-}\right)^{-l-1} \widetilde{\boldsymbol{a}}_{1, k}^{\mathrm{ref},(0),-} \\
& \psi_{-1, k}^{\text {in }}=\left(\lambda_{L, k}^{+}\right)^{-l-1} \phi_{L, k}^{+} \text {. }
\end{aligned}
$$

Hereafter, we will leave out the symbols + and - because it is obvious which generalized Bloch waves are included in the SWFs when the index of the layer is indicated. In addition, the index of the incident waves $k$ will be also omitted for notational simplicity.

\section{Boundary condition}

Table I summarizes the expressions for the electrode regions and transition region in the WFM methods proposed so far. The electrode regions are the layers where the transmitted and reflected waves are defined by a linear combination of generalized Bloch waves as in Eqs. (7) and (9), respectively. The transition region corresponds to the layers in which the values on real-space grids or coefficients for the atomic bases of the SWFs are calculated using the WFM procedure. In the previous WFM methods, the values or coefficients at the edge layers of the transition region are defined on real-space grids or expanded using an atomic basis set, whereas they are given by a linear combination of generalized Bloch waves as a boundary condition in the electrode regions [e.g., Eqs. (7) and (9)]. As long as the complete set of generalized Bloch waves is employed, the SWFs determined in the electrode and transition regions are identical. However, numerical errors will occur when the rapidly varying evanescent waves are excluded by the cutoff parameter of the Bloch factor $\lambda_{\text {min }}$. In our procedure, to keep numerical rigorousness, the overlap of the layers between the electrode and transition regions is eliminated.

\section{E. Moving matching plane of wave function matching formula}

In Appendix B, the WFM formula [11] for the case that the rank of $\overline{\mathbf{H}}_{L}\left(\overline{\mathbf{H}}_{R}\right), m_{L}\left(m_{R}\right)$ and the rank of $\overline{\mathbf{H}}_{L, L}\left(\overline{\mathbf{H}}_{R, R}\right)$, $m_{L L}\left(m_{R R}\right)$ are equal is derived. Following the procedure in Appendix B, the scheme to move the matching plane is introduced in this section. For the case that the rank of $\overline{\mathbf{H}}_{L, L}\left(\overline{\mathbf{H}}_{R, R}\right)$, 
$m_{L L}\left(m_{R R}\right)$ are not equal is discussed in Appendix C. By letting $\widehat{\mathbf{H}}^{(0)}$ be the $m_{T}$-dimensional Hamiltonian for the computational model without any extra layers [Fig. 1(a)], the Hamiltonian $\widehat{\mathbf{H}}^{(l)}$ and overlap $\widehat{\mathbf{S}}^{(l)}$ with $l$ extra layers [Fig. 1(b)] are described by $\left[m_{T}+l\left(m_{L}+m_{R}\right)\right]$-dimensional matrices. Using $\widehat{\mathbf{H}}^{(l)}$ and $\widehat{\mathbf{S}}^{(l)}$, the Green's function of the isolated transition region is expressed as $\widehat{\mathbf{G}}^{(l)}\left[=\left(E \widehat{\mathbf{S}}^{(l)}-\widehat{\mathbf{H}}^{(l)}\right)^{-1}\right]$. As introduced in Appendix B, the WFM formula is determined as

$$
\left(\begin{array}{cc}
-\mathbf{G}_{1-l, 1-l}^{(l)} \overline{\mathbf{H}}_{L, L}^{\dagger}-\left(\mathbf{R}^{\mathrm{ref},(l)}\right)^{-1} & -\mathbf{G}_{1-l, n+l}^{(l)} \overline{\mathbf{H}}_{R, R} \\
-\mathbf{G}_{n+l, 1-l}^{(l)} \overline{\mathbf{H}}_{L, L}^{\dagger} & -\mathbf{G}_{n+l, n+l}^{(l)} \overline{\mathbf{H}}_{R, R}-\left(\mathbf{R}^{\mathrm{raa},(l+1)}\right)^{-1}
\end{array}\right)\left(\begin{array}{c}
\psi_{0}^{\mathrm{ref}} \\
\psi_{n+1}
\end{array}\right)=\left(\begin{array}{c}
\mathbf{G}_{1-l, 1-l}^{(l)} \overline{\mathbf{H}}_{L, L}^{\dagger} \psi_{0}^{\mathrm{in}}+\psi_{1}^{\mathrm{in},(l)} \\
\mathbf{G}_{n+l, 1-l}^{(l)} \overline{\mathbf{H}}_{L, L}^{\dagger} \psi_{0}^{\mathrm{in}}
\end{array}\right),
$$

where $\quad \mathbf{R}^{\mathrm{ref},(l)}=\psi_{0}^{\mathrm{ref}}\left(\psi_{1}^{\mathrm{ref},(l)}\right)^{-1}, \quad \mathbf{R}^{\mathrm{tra},(l+1)}=\psi_{n+1}\left(\psi_{n}^{(l)}\right)^{-1}$, $\mathbf{G}_{i, j}^{(l)}$ is the $(i, j)$ th block-matrix element of the Green's function of the isolated transition region $\widehat{\mathbf{G}}^{(l)}$, which is introduced by Eq. (B3) in Appendix B. The ratio matrices $\mathbf{R}^{\text {ref, }(l)}$ and $\mathbf{R}^{\text {tra, }(l+1)}$ are obtained by solving the continued fraction equations

$$
\begin{gathered}
\mathbf{R}^{\mathrm{ref},(l-1)}=-\left(\overline{\mathbf{H}}_{L}+\overline{\mathbf{H}}_{L, L}^{\dagger} \mathbf{R}^{\mathrm{ref},(l)}\right)^{-1} \overline{\mathbf{H}}_{L, L}, \\
\mathbf{R}^{\operatorname{tra},(l)}=-\left(\overline{\mathbf{H}}_{R}+\overline{\mathbf{H}}_{R, R} \mathbf{R}^{\operatorname{tra},(l+1)}\right)^{-1} \overline{\mathbf{H}}_{R, R}^{\dagger}
\end{gathered}
$$

using the generalized Bloch waves at the deep inside of electrodes.

The extension of the transition region requires additional computations because the number of dimensions of the Green's functions $\widehat{\mathbf{G}}^{(l)}$ linearly increase with respect to the number of inserted extra layers. In this subsection, we describe the procedure to move the matching planes to the inside of the transition region without loss of accuracy. In Appendix B, the left (right) matching plane is set between $\psi_{0}$ and $\psi_{1}^{(l)}\left(\psi_{n}^{(l)}\right.$ and $\left.\psi_{n+1}\right)$. We are going to set the left (right) matching plane between $\psi_{1}^{(l)}$ and $\psi_{1}^{(l-1)}\left(\psi_{n}^{(l-1)}\right.$ and $\left.\psi_{n}^{(l-1)}\right)$. Khomyakov et al. proved the translational invariance of the total transmission probability with respect to moving the matching planes to the inside of the electrode regions [13]. However, when the matching planes are moved to the inside of the transition region, we need to treat the rapidly decreasing evanescent waves, which are excluded from the electrode regions by introducing the cutoff parameter $\lambda_{\text {min }}$ but contribute to the SWFs in the transition region.

Using the Kohn-Sham Hamiltonian for the model with $l-1$ extra layers, the Hamiltonian for the model with $l$ extra layers can be rewritten as

$$
\begin{aligned}
& E \widehat{\mathbf{S}}^{(l)}-\widehat{\mathbf{H}}^{(l)} \\
& =\left(\begin{array}{ccc}
E \mathbf{S}_{L}-\mathbf{H}_{L} & \mathbf{A}_{L}^{(l)} & \mathbf{0} \\
\mathbf{A}_{L}^{(l) \dagger} & E \widehat{\mathbf{S}}^{(l-1)}-\widehat{\mathbf{H}}^{(l-1)} & \mathbf{A}_{R}^{(l)} \\
\mathbf{0} & \mathbf{A}_{R}^{(l) \dagger} & E \mathbf{S}_{R}-\mathbf{H}_{R}
\end{array}\right) \\
& =\left(\begin{array}{ccc}
\overline{\mathbf{H}}_{L} & \mathbf{A}_{L}^{(l)} & \mathbf{0} \\
\mathbf{A}_{L}^{(l) \dagger} & E \widehat{\mathbf{S}}^{(l-1)}-\widehat{\mathbf{H}}^{(l-1)} & \mathbf{A}_{R}^{(l)} \\
\mathbf{0} & \mathbf{A}_{R}^{(l) \dagger} & \overline{\mathbf{H}}_{R}
\end{array}\right),
\end{aligned}
$$

where

$$
\begin{aligned}
& \mathbf{A}_{L}^{(l)}=\left(\overline{\mathbf{H}}_{L, L}, \mathbf{0}, \ldots, \mathbf{0}\right), \\
& \mathbf{A}_{R}^{(l)}=\left(\overline{\mathbf{H}}_{R, R}, \mathbf{0}, \ldots, \mathbf{0}\right) .
\end{aligned}
$$

$\mathbf{A}_{L}^{(l)}\left(\mathbf{A}_{R}^{(l)}\right)$ is the $m_{L} \times\left(m_{T}+(l-1)\left(m_{L}+m_{R}\right)\right)\left[m_{R} \times\left(m_{T}+\right.\right.$ $\left.\left.(l-1)\left(m_{L}+m_{R}\right)\right)\right]$ zero matrix except that the $m_{L} \times$ $m_{L}\left(m_{R} \times m_{R}\right)$ block-matrix element at the left edge is $\overline{\mathbf{H}}_{L, L}\left(\overline{\mathbf{H}}_{R, R}\right)$. Consequently, Eq. (B1) becomes

$$
\begin{aligned}
& \left(\begin{array}{c|c|c}
\overline{\mathbf{H}}_{L} & \mathbf{A}_{L}^{(l)} & \mathbf{0} \\
\mathbf{A}_{L}^{(l) \dagger} & E \widehat{\mathbf{S}}^{(l-1)}-\widehat{\mathbf{H}}^{(l-1)} & \mathbf{A}_{R}^{(l)} \\
\mathbf{0} & \mathbf{A}_{R}^{(l) \dagger} & \overline{\mathbf{H}}_{R}
\end{array}\right)\left(\begin{array}{c}
\frac{\psi_{1}^{(l)}}{\psi_{1}^{(l-1)}} \\
\vdots \\
\frac{\psi_{n}^{(l-1)}}{\psi_{n}^{(l)}}
\end{array}\right) \\
& =\left(\begin{array}{c}
-\overline{\mathbf{H}}_{L, L}^{\dagger} \psi_{0} \\
0 \\
\vdots \\
0 \\
\frac{0}{-\overline{\mathbf{H}}_{R, R} \psi_{n+1}}
\end{array}\right) .
\end{aligned}
$$

Eliminating the first and last block rows yields

$$
\left(E \widehat{\mathbf{S}}^{(l-1)}-\widehat{\mathbf{H}}^{(l-1)}\right)\left(\begin{array}{c}
\psi_{1}^{(l-1)} \\
\vdots \\
\vdots \\
\vdots \\
\psi_{n}^{(l-1)}
\end{array}\right)=\left(\begin{array}{c}
-\overline{\mathbf{H}}_{L, L}^{\dagger} \psi_{1}^{(l)} \\
0 \\
\vdots \\
0 \\
-\overline{\mathbf{H}}_{R, R} \psi_{n}^{(l)}
\end{array}\right) .
$$

Using $\widehat{\mathbf{G}}^{(l-1)}$, Eq. (33) can be rewritten as

$$
\left(\begin{array}{c}
\psi_{1}^{(l-1)} \\
\vdots \\
\vdots \\
\vdots \\
\psi_{n}^{(l-1)}
\end{array}\right)=\widehat{\mathbf{G}}^{(l-1)}\left(\begin{array}{c}
-\overline{\mathbf{H}}_{L, L}^{\dagger} \psi_{1}^{(l)} \\
0 \\
\vdots \\
0 \\
-\overline{\mathbf{H}}_{R, R} \psi_{n}^{(l)}
\end{array}\right) \text {. }
$$

In analogy to Eq. (B1), we then have a WFM formula where the matching planes are shifted by one layer inside of the transition region.

$$
\left(\begin{array}{cc}
-\mathbf{G}_{2-l, 2-l}^{(l-1)} \overline{\mathbf{H}}_{L, L}^{\dagger}-\left(\mathbf{R}^{\mathrm{ref},(l-1)}\right)^{-1} & -\mathbf{G}_{2-l, n+l-1}^{(l-1)} \overline{\mathbf{H}}_{R, R} \\
-\mathbf{G}_{n+l-1,2-l}^{(l-1)} \overline{\mathbf{H}}_{L, L}^{\dagger} & -\mathbf{G}_{n+l-1, n+l-1}^{(l-1)} \overline{\mathbf{H}}_{R, R}-\left(\mathbf{R}^{\mathrm{tra},(l)}\right)^{-1}
\end{array}\right)\left(\begin{array}{c}
\psi_{1}^{\mathrm{ref},(l)} \\
\psi_{n}^{(l)}
\end{array}\right)=\left(\begin{array}{c}
\mathbf{G}_{2-l, 2-l}^{(l-1)} \overline{\mathbf{H}}_{L, L}^{\dagger} \psi_{1}^{\mathrm{in},(l)}+\psi_{1}^{\mathrm{in},(l-1)} \\
\mathbf{G}_{n+l-1,2-l}^{(l-1)} \overline{\mathbf{H}}_{L, L}^{\dagger} \psi_{1}^{\mathrm{in},(l)}
\end{array}\right) .
$$


Substituting $\mathbf{R}^{\text {ref, }(l)}$ and $\mathbf{R}^{\text {tra, }(l+1)}$ into Eqs. (27) and (28), respectively, we obtain $\mathbf{R}^{\text {ref, }(l-1)}$ and $\mathbf{R}^{\text {tra, }(l)}$. Although the Green's function $\widehat{\mathbf{G}}^{(l-1)}$ is required, its dimension is reduced from $m_{T}+l\left(m_{L}+m_{R}\right)$ to $m_{T}+(l-1)\left(m_{L}+m_{R}\right)$.

By repeating the shift of the matching planes by using Eqs. (26) and (35) and solving Eqs. (27) and (28) $l$ times, the matching

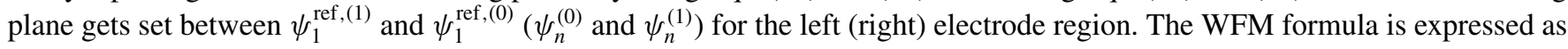

$$
\left(\begin{array}{cc}
-\mathbf{G}_{1,1}^{(0)} \overline{\mathbf{H}}_{L, L}^{\dagger}-\left(\mathbf{R}^{\mathrm{ref},(0)}\right)^{-1} & -\mathbf{G}_{1, n}^{(0)} \overline{\mathbf{H}}_{R, R} \\
-\mathbf{G}_{n, 1}^{(0)} \overline{\mathbf{H}}_{L, L}^{\dagger} & -\mathbf{G}_{n, n}^{(0)} \overline{\mathbf{H}}_{R, R}-\left(\mathbf{R}^{\mathrm{tra},(1)}\right)^{-1}
\end{array}\right)\left(\begin{array}{c}
\psi_{1}^{\mathrm{ref},(1)} \\
\psi_{n}^{(1)}
\end{array}\right)=\left(\begin{array}{c}
\mathbf{G}_{1,1}^{(0)} \overline{\mathbf{H}}_{L, L}^{\dagger} \psi_{1}^{\mathrm{in},(1)}+\psi_{1}^{\mathrm{in},(0)} \\
\mathbf{G}_{n, 1}^{(0)} \overline{\mathbf{H}}_{L, L}^{\dagger} \psi_{1}^{\mathrm{in},(1)}
\end{array}\right) .
$$

The SWFs in the transition region are computed by substituting $\psi_{1}^{\text {ref,(1) }}$ and $\psi_{n}^{(1)}$ into Eq. (34).

This result implies that the translational invariance of the SWFs with respect to moving the matching planes to the inside of the transition region is preserved even when the rapidly varying evanescent waves are excluded. In addition, when the sum of the number of iterations for Eqs. (27) and (28) and the number of the extra layers are identical, exactly the same SWFs can be obtained as long as the boundary condition is properly imposed, as mentioned in Sec. IID. In Sec. III A, we demonstrate that the translational invariance is not retained when the transition region and electrode regions overlap as shown in Table I and the set of generalized Bloch waves is incomplete.

Finally, let us consider the convergence of Eqs. (27) and (28). Suppose that the solutions of Eq. (36) with $l$ extra layers are $\psi_{1}^{\text {ref,(1) }}$ and $\psi_{n}^{(1)}$ and the solutions with $l+1$ extra layers are $\psi_{1}^{\prime \text { ref,(1) }}$ and $\psi_{n}^{\prime(1)}, \psi_{1}^{\text {ref,(1) }}=\psi_{1}^{\text {'ref,(1) }}$ and $\psi_{n}^{(1)}=\psi_{n}^{\prime(1)}$ when $l$ is large enough. The first row of Eq. (36) for $l$ extra layers is

$$
\begin{aligned}
& -\mathbf{G}_{1,1}^{(0)} \overline{\mathbf{H}}_{L, L}^{\dagger} \psi_{1}^{\mathrm{ref},(1)}-\left(\mathbf{R}^{\mathrm{ref},(0)}\right)^{-1} \psi_{1}^{\mathrm{ref},(1)}-\mathbf{G}_{1, n}^{(0)} \overline{\mathbf{H}}_{R, R} \psi_{n}^{(1)} \\
& =\mathbf{G}_{1,1}^{(0)} \overline{\mathbf{H}}_{L, L}^{\dagger} \psi_{1}^{\mathrm{in},(1)}+\psi_{1}^{\mathrm{in},(0)} .
\end{aligned}
$$

Inserting $\psi_{1}^{\text {ref,(1) }}$ and $\psi_{n}^{(1)}$ into Eq. (36) for $l+1$ extra layers, we have

$$
\begin{aligned}
& -\mathbf{G}_{1,1}^{(0)} \overline{\mathbf{H}}_{L, L}^{\dagger} \psi_{1}^{\mathrm{ref},(1)}-\left(\mathbf{R}^{\prime \mathrm{ref},(0)}\right)^{-1} \psi_{1}^{\mathrm{ref},(1)}-\mathbf{G}_{1, n}^{(0)} \overline{\mathbf{H}}_{R, R} \psi_{n}^{(1)} \\
& =\mathbf{G}_{1,1}^{(0)} \overline{\mathbf{H}}_{L, L}^{\dagger} \psi_{1}^{\mathrm{in},(1)}+\psi_{1}^{\mathrm{in},(0)}
\end{aligned}
$$

where $\mathbf{R}^{\prime \text { ref,(0) }}$ is the ratio matrix of the computational model with $l+1$ extra layers. Subtracting Eq. (38) from Eq. (37), we obtain

$$
\left(\mathbf{R}^{\text {ref, }(0)}\right)^{-1}=\left(\mathbf{R}^{\prime \text { ref, }(0)}\right)^{-1} .
$$

$\mathbf{R}^{\text {ref,(0) }}$ is computed by solving Eq. (27) $l+1$ times with the initial matrix of $\left(\widetilde{\boldsymbol{\Lambda}}_{L}^{-}\right)^{-1} \widetilde{\boldsymbol{\Phi}}_{L}^{-}\left(\widetilde{\boldsymbol{\Phi}}_{L}^{-}\right)^{-1}$, while $\mathbf{R}^{\prime \text { ref,(0) }}$ is obtained by solving Eq. (27) $l+2$ times with the same initial matrix. This indicates that $\mathbf{R}^{\text {ref,(0) }}$ is uniquely determined when $l$ is sufficiently large. In other words, by repeatedly solving Eqs. (27) and (28) until $\mathbf{R}^{\text {ref,(0) }}$ and $\mathbf{R}^{\text {tra,(1) }}$ become consistent, we obtain $\mathbf{R}^{\text {ref,(0) }}$ and $\mathbf{R}^{\text {tra,(1) }}$ for $l \rightarrow \infty$ and the SWFs for a truly semi-infinite system. The convergence behavior of the ratio matrix is demonstrated in Appendix D.

\section{F. Transmission probability}

The expression of the conductance at zero bias limit is given by the Landauer-Büttiker formula [17]. The Landauer-Büttiker formula using the transmission coefficient matrix is

$$
G(E)=\frac{2 e^{2}}{h} \operatorname{Tr} \mathbf{T}=\frac{2 e^{2}}{h} \operatorname{Tr}\left[\left(\mathbf{v}^{\text {in }}\right)^{-1} \mathbf{t}^{\dagger} \mathbf{v}^{\operatorname{tra}} \mathbf{t}\right],
$$

where $\mathbf{t}$ is an $m_{R R} \times m_{k}$ transmission coefficient matrix with $m_{k}$ being the number of the incident waves, and $\mathbf{v}^{\text {in }}\left(\mathbf{v}^{\text {tra }}\right)$ is an $m_{k}-\left(m_{R R^{-}}\right)$dimensional matrix describing the group velocity of the incident (transmission) waves. In Ref. [14], the group velocity of the incident waves is written as

$$
\mathbf{v}^{\text {in }}=a \boldsymbol{\Phi}_{L}^{\text {in, }+\dagger} \boldsymbol{\Gamma}_{L}^{(0)} \boldsymbol{\Phi}_{L}^{\text {in, },},
$$

where

$$
\boldsymbol{\Phi}_{L}^{\text {in, }+}=\left\{\phi_{L, 1}^{+}, \ldots, \phi_{L, m_{k}}^{+}\right\} .
$$

The matrix for the group velocity is diagonal when the selfenergy terms of electrodes are exactly obtained. However, due to the exclusion of the rapidly varying evanescent waves and numerical error in the computation of the Green's functions, the off-diagonal elements do not vanish completely. The group velocity of the transmitted waves is expressed as

$$
\mathbf{v}^{\text {tra }}=a\left(\widetilde{\boldsymbol{\Phi}}_{R}^{+}\right)^{\dagger} \boldsymbol{\Gamma}_{R}^{(0)} \widetilde{\boldsymbol{\Phi}}_{R}^{+} .
$$

Here, $a$ is the length of the unit cell and

$$
\Gamma_{L}^{(l)}=\mathrm{i}\left(\Sigma_{L}^{(l)}-\Sigma_{L}^{(l) \dagger}\right),
$$

where $\Sigma_{L}^{(l)}$ and $\Sigma_{R}^{(l)}$ are the self-energy terms of the electrodes, which are expressed as

$$
\begin{gathered}
\Sigma_{L}^{(l)}=-\overline{\mathbf{H}}_{L, L}^{\dagger} \mathbf{R}^{\mathrm{ref},(l)}, \\
\Sigma_{R}^{(l)}=-\overline{\mathbf{H}}_{R, R} \mathbf{R}^{\mathrm{tra},(l+1)} .
\end{gathered}
$$

From Eq. (18), the transmission coefficient matrix is obtained by

$$
\boldsymbol{t}_{k}=\left(\widetilde{\boldsymbol{\Phi}}_{R}^{+}, \stackrel{\circ}{\mathbf{\Phi}}_{R}^{+}\right)^{-1} \psi_{n, k}^{(1)} \approx\left(\widetilde{\boldsymbol{\Phi}}_{R}^{+}, \stackrel{\circ}{\mathbf{0}}\right)^{-1} \psi_{n, k}^{(1)},
$$

and

$$
\mathbf{t}=\left(\boldsymbol{t}_{1}, \ldots, \boldsymbol{t}_{m_{k}}\right) .
$$

Khomyakov et al. and Sørensen et al. instructed one to use the pseudoinverses for $\left(\widetilde{\boldsymbol{\Phi}}_{R}^{+}, \mathbf{0}\right)[13,18]$. However, the SWFs $\psi_{n, k}^{(1)}$ include the components of $\dot{\boldsymbol{\Phi}}_{R}^{+}$, because they are determined on real-space grids or linear combinations of bases in the transition region. In addition, as we show in Appendix E, translational invariance is not retained when the pseudoinverses are used in the computation of the transmission coefficients in Eq. (47). Therefore, we introduce $\boldsymbol{\Phi}_{R}^{\prime}$ so that

$$
\check{\boldsymbol{\Phi}}_{R}^{+}=\left(\widetilde{\boldsymbol{\Phi}}_{R}^{+}, \boldsymbol{\Phi}_{R}^{\prime}\right)
$$


becomes a regular matrix. Using $\check{\boldsymbol{\Phi}}_{R}^{+}$, we define

$$
\begin{aligned}
\check{\mathbf{v}}^{\text {tra }} & =a\left(\check{\boldsymbol{\Phi}}_{R}^{+}\right)^{\dagger} \boldsymbol{\Gamma}_{R}^{(0)} \check{\boldsymbol{\Phi}}_{R}^{+}, \\
\check{\boldsymbol{t}}_{k} & =\left(\check{\boldsymbol{\Phi}}_{R}^{+}\right)^{-1} \psi_{n, k}^{(1)}, \\
\check{\mathbf{t}} & =\left(\check{\boldsymbol{t}}_{1}, \ldots, \check{\boldsymbol{t}}_{m_{k}}\right) .
\end{aligned}
$$

Substituting Eqs. (50)-(52), we have

$$
\mathbf{T}=\left(\mathbf{v}^{\text {in }}\right)^{-1} \check{\mathbf{t}}^{\dagger} \check{\mathbf{v}}^{\text {tra } \check{\mathbf{t}}}=\left(\mathbf{v}^{\text {in }}\right)^{-1} \mathbf{t}^{\dagger} \mathbf{v}^{\text {tra }} \mathbf{t} .
$$

The transmission matrix is invariant when one takes $\boldsymbol{\Phi}_{R}^{\prime}$ so that $\check{\boldsymbol{\Phi}}_{R}^{+}$is a regular matrix. The proof is given in Appendix F. Very often one is only interested in the conductance. In that case one can skip the computation of the transmission coefficients. The $k$ th diagonal element of the transmission matrix $T_{k, k}$ is given by

$$
\begin{aligned}
T_{k, k} & =\left(v_{k, k}^{\text {in }}\right)^{-1} \check{\boldsymbol{t}}_{k}^{\dagger} \check{\mathbf{v}}^{\operatorname{tra}} \check{\boldsymbol{t}}_{k} \\
& =a\left(v_{k, k}^{\mathrm{in}}\right)^{-1}\left[\left(\check{\boldsymbol{\Phi}}_{R}^{+}\right)^{-1} \psi_{n, k}^{(1)}\right]^{\dagger}\left(\check{\boldsymbol{\Phi}}_{R}^{+}\right)^{\dagger} \boldsymbol{\Gamma}_{R}^{(0)} \check{\boldsymbol{\Phi}}_{R}^{+}\left(\check{\boldsymbol{\Phi}}_{R}^{+}\right)^{-1} \psi_{n, k}^{(1)} \\
& =a\left(v_{k, k}^{\mathrm{in}}\right)^{-1} \psi_{n, k}^{(1) \dagger} \boldsymbol{\Gamma}_{R}^{(0)} \psi_{n, k}^{(1)},
\end{aligned}
$$

where $v_{i, j}^{\text {in }}$ is the $(i, j)$ th element of $\mathbf{v}^{\text {in }}$. Analogous to the transmission matrix and probability, the reflection matrix and probability can be obtained from $\psi_{1, k}^{\text {ref,(1) }}$. Note that the transmission probability is given only from $\psi_{n, k}^{(1)}$ and $\boldsymbol{\Gamma}_{R}^{(0)}$. This is contrastive to the nonequilibrium Green's function method, which uses the Green's function of the transition region being expensive to be calculated. Section III B describes the efficiency of using the inverse of a regular matrix $\check{\boldsymbol{\Phi}}_{R}^{+}$ instead of the pseudoinverse of $\widetilde{\boldsymbol{\Phi}}_{R}^{+}$.

\section{NUMERICAL EXAMPLE}

\section{A. Translational invariance of transmission probability with respect to moving matching plane}

Sørensen et al. proposed a method that extends the transition region by inserting extra layers to improve the accuracy of the WFM technique [18]. Insertion of the extra layers corresponds to moving the matching plane to the inside of the electrode region. From the problem of the penetration of quantum particles into a one-dimensional square potential barrier on the basis of quantum mechanics, it is obvious that the transmission probability does not depend on the position of the matching plane. Translational invariance is retained as long as all the generalized Bloch waves are included for the WFM procedure. When the rapidly decreasing evanescent waves are excluded by the cutoff parameter $\lambda_{\min }$, it is not trivial whether the invariance is kept at the boundary between the electrode and transition regions. Khomyakov et al. proved the translational invariance with respect to moving the matching plane toward the electrode region [13]. Moving the matching plane to the electrode side is straightforward because the excluded evanescent waves do not contribute to the SWFs anymore. When the planes are moved into the transition region, one needs to consider the contribution of the excluded evanescent waves because the waves do not vanish in the transition region.

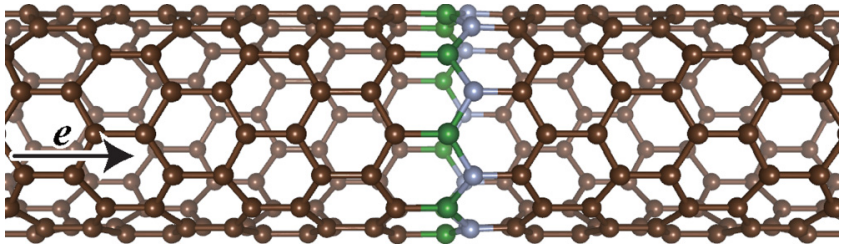

FIG. 2. Computational model where one B-N zigzag ring is sandwiched between a couple of $(9,0) \mathrm{CNT}$ electrodes. The light blue, dark brown, and green balls are $\mathrm{N}, \mathrm{C}$, and B atoms, respectively.

As an example of a practical calculation, we will examine the transport properties of a $(9,0) \mathrm{B}-\mathrm{N}$ nanotube sandwiched between $(9,0)$ carbon nanotubes (CNTs). Figure 2 shows the computational model. The $x$ and $y$ axes are the directions perpendicular to the nanotube, and the $z$ axis is parallel to the nanotube. A valence electron-ion interaction is treated by norm-conserving pseudopotentials generated by the scheme proposed by Troullier and Martins [23,29]. The local density approximation [30] of the density functional theory $[31,32]$ is used to describe the exchange and correlation effects. We use the real-space finite-difference method for the first-principles calculation implemented in the first-principles calculation code RSPACE [12,33-35], which enables us to calculate the transport properties of nanostructures between the semi-infinite electrodes. In the real-space grid formalism, $m_{L}\left(m_{R}\right)$ is not equal to $m_{L L}\left(m_{R R}\right)$. For that reason, the procedure described in Appendix $C$ is used. The central finite-difference formula [36] ( $N=1$ in Ref. [37]) is used for the second-order derivation arising from the kinetic-energy operator in the Kohn-Sham equation. A conventional supercell under periodic boundary conditions in all directions with a real-space grid spacing of $\sim 0.24 \AA$ is used to determine the Kohn-Sham effective potential. The dimensions of the supercell are set as $L_{x}=$ $L_{y}=13.34 \AA$, and $L_{z}=4.32 \AA$ for the electrode regions and $L_{x}=L_{y}=13.34 \AA$, and $L_{z}=8.64 \AA$ for the transition region. Here, $L_{x}$ and $L_{y}$ are the lateral lengths of the supercell in the $x$ and $y$ directions, respectively, and $L_{z}$ is the length in the $z$ direction. Propagating and slowly decaying evanescent states of the semi-infinite electrodes are calculated using the method in Ref. [24]. Table II shows the transmission probability with respect to the number of extra layers $l$ and the number of iterations for the continued-fraction equations [Eqs. (C19) and (C20)] $N_{i t}$. Since all the WFM methods proposed so far use the same boundary conditions between the electrode and transition regions, we use the method proposed by Fujimoto and Hirose [11]. To eliminate the numerical error caused by the pseudoinverses, all the generalized Bloch waves are included in $\widetilde{\boldsymbol{\Phi}}_{R}^{+}$for Eq. (47). In the conventional WFM formalisms, the computation of the continued-fraction equations is skipped when the extra layer is not inserted because $\psi_{1}^{\text {ref, },(l)}\left(\xi_{1}\right)\left(\psi_{0}^{\text {ref }}\left(\xi_{3}\right)\right)^{-1}$ and $\psi_{n}^{(l)}\left(\xi_{3}\right)\left(\psi_{n+1}\left(\xi_{1}\right)\right)^{-1}$ in Eq. (C5) can be obtained as $\widetilde{\boldsymbol{\Phi}}_{L}^{-}\left(\xi_{1}\right)\left(\left(\widetilde{\boldsymbol{\Lambda}}_{L}^{-}\right)^{-1} \widetilde{\boldsymbol{\Phi}}_{L}^{-}\left(\xi_{3}\right)\right)^{-1}$ and $\widetilde{\boldsymbol{\Phi}}_{R}^{+}\left(\xi_{3}\right)\left(\widetilde{\boldsymbol{\Lambda}}_{R}^{+} \widetilde{\boldsymbol{\Phi}}_{R}^{+}\left(\xi_{1}\right)\right)^{-1}$, respectively, owing to the overlap of the layers in the transition and electrode regions. One can see that while the translational invariance deteriorates between $N_{i t}=0$ and $N_{i t}=1$ in the conventional WFM methods, it is retained nicely in the present method. The translational invariance is 
TABLE II. Transmission probability with respect to the number of extra layers $l$ and number of iterations for the continued-fraction equations $N_{i t}$ for $(9,0)$ B-N nanotube between $(9,0)$ CNTs.

\begin{tabular}{ccccc}
\hline \hline$\lambda_{\text {min }}$ & $l$ & $N_{i t}$ & Conventional method & Present method \\
\hline 0.90 & 3 & 0 & 0.52046191 & 0.52046575 \\
& 2 & 1 & 0.52045862 & 0.52046575 \\
& 1 & 2 & 0.52045862 & 0.52046575 \\
& 0 & 3 & 0.52045862 & 0.52046575 \\
& 2 & 0 & 0.52044949 & 0.52045862 \\
& 1 & 1 & 0.52044135 & 0.52045862 \\
& 0 & 2 & 0.52044135 & 0.52045862 \\
& 1 & 0 & 0.52041587 & 0.52044135 \\
0.65 & 0 & 1 & 0.52039468 & 0.52044135 \\
& 3 & 0 & 0.52046511 & 0.52046809 \\
& 2 & 1 & 0.52046378 & 0.52046809 \\
& 1 & 2 & 0.52046378 & 0.52046809 \\
& 0 & 3 & 0.52046378 & 0.52046809 \\
& 2 & 0 & 0.52045659 & 0.52046378 \\
& 1 & 1 & 0.52045277 & 0.52046378 \\
& 0 & 2 & 0.52045277 & 0.52046378 \\
& 1 & 0 & 0.52043133 & 0.52045277 \\
0.10 & 0 & 1 & 0.52042062 & 0.52045277 \\
& 2 & 0 & 0.52047069 & 0.52047070 \\
& 1 & 1 & 0.52047070 & 0.52047070 \\
& 0 & 2 & 0.52047070 & 0.52047070 \\
& 1 & 0 & 0.52046908 & 0.52047070 \\
& 0 & 1 & 0.52047028 & 0.52047070 \\
\hline \hline & & & & \\
& & & & \\
6
\end{tabular}

destroyed by the inconsistency of the SWFs at the overlapping layers; the deterioration is suppressed when $\lambda_{\min }$ is small or $l$ is large.

From these results, we can conclude that the overlap between the electrode and transition region should be removed to maintain the translational invariance of the transmission probability. However, the numerical error caused by the deterioration of the translational invariance of the transmission probability is small and is not the origin of the degradation of the accuracy when the transition layer is not extended as reported in Ref. [18].

\section{B. Accuracy of the transmission matrix and probability obtained by SWF}

In Sec. IIF, we described a method for calculating the transmission and reflection probabilities without the pseudoinverses. Here, to demonstrate the accuracy of this method and the degradation due to the pseudoinverses, we examine the variations in the transport properties with respect to the number of extra layers $l$ and cutoff parameter of the evanescent waves $\lambda_{\text {min }}$. The computational model is the same as in Sec. III A. To eliminate the unfavorable effect from the convergence of the ratio matrices on the SWFs, the ratio matrices are converged using the continued-fraction equations Eqs. (C19) and (C20). The behaviors of the transmission probability, transmission matrix, and the sum of the transmission and reflection probabilities with respect to the number of extra layers $l$ are plotted in Fig. 3 . We can see that the translational invariances of the transmission probability and transmission matrix are well preserved and the sum of the probabilities exactly corresponds with the number
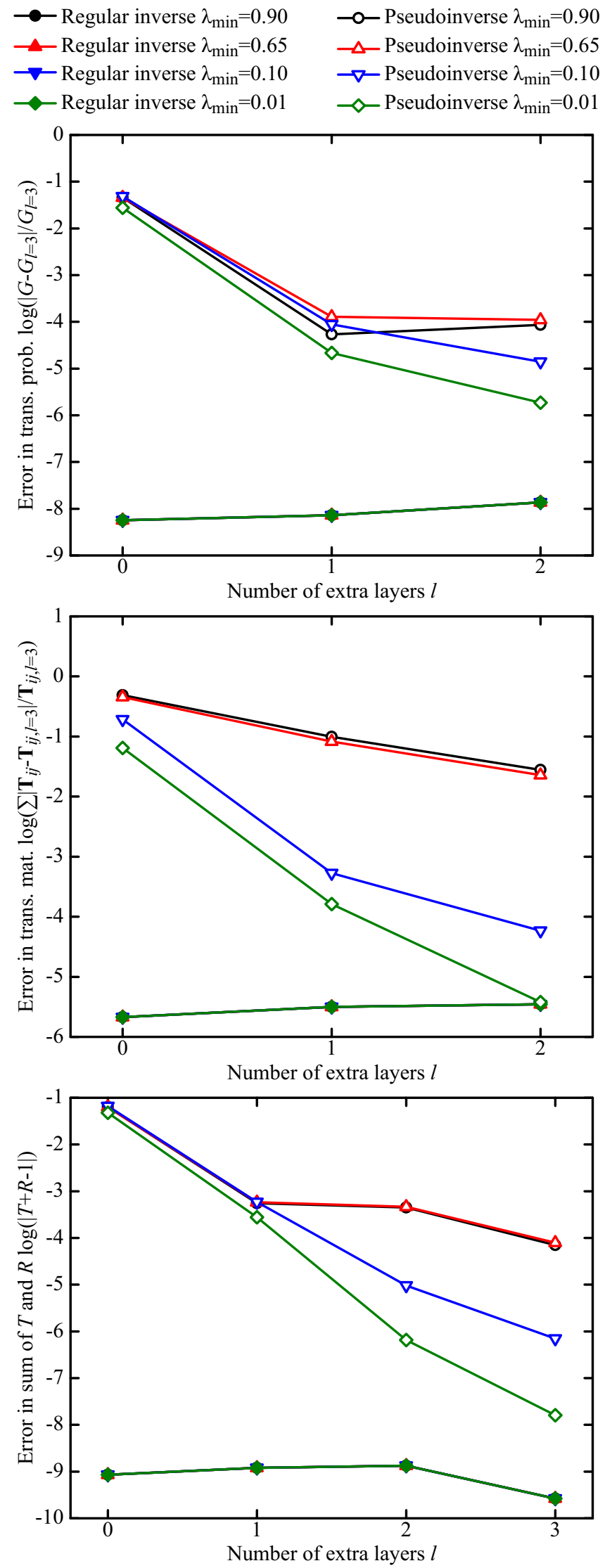

FIG. 3. Difference in transmission probability and matrix and error in the sum of the transmission and reflection probabilities with respect to the number of extra layers $l$. 


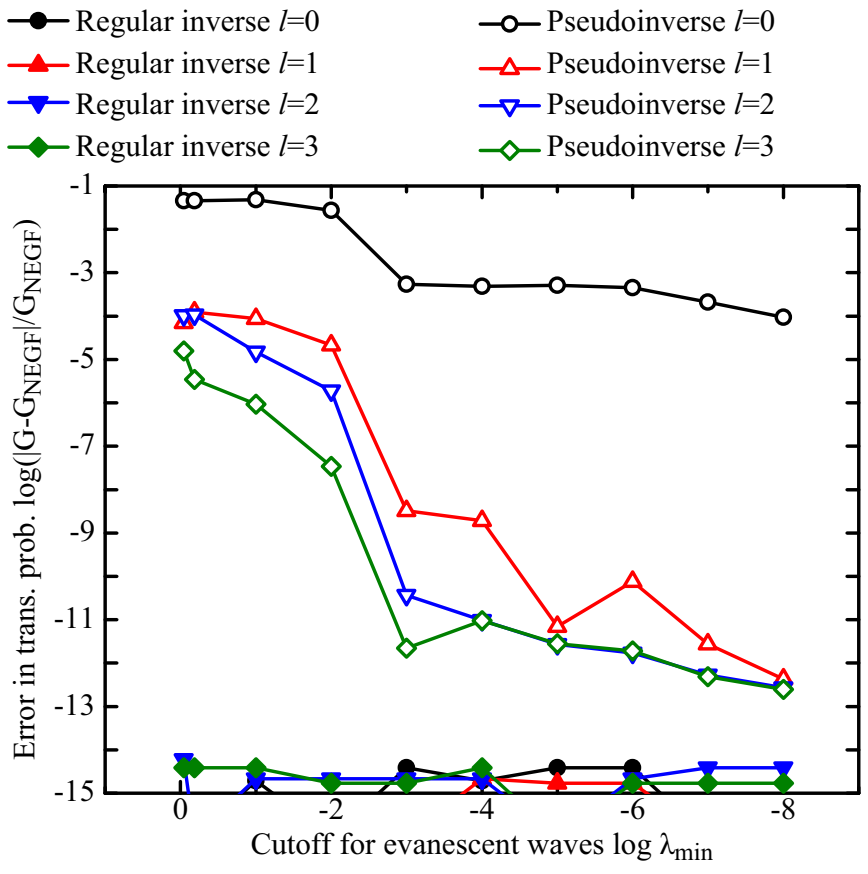

FIG. 4. Difference in the transmission probability from that obtained by the nonequilibrium Green's function method with respect to the cutoff parameter of the evanescent waves $\lambda_{\min }$.

of channels in the present method. On the other hand, the transmission probability and transmission matrix probability are clearly affected by the number of extra layers when the pseudoinverses are used. Moreover, the sum of the transmission and reflection probabilities does not correspond to the number of channels, indicating that the pseudoinverses degrade the accuracy. Figure 4 shows the difference in the transmission probability from that obtained by the nonequilibrium Green's function method with respect to the cutoff parameter $\lambda_{\text {min }}$ of the evanescent waves. We can conclude that the accuracy of the WFM technique on the transmission probability is comparable with that of the nonequilibrium Green's function method even when the rapidly varying evanescent waves $\left(|\lambda|<\lambda_{\min }\right.$ or $\left.1 / \lambda_{\min }<|\lambda|\right)$ are not explicitly computed and the transition region is not extended.

When transmission spectra are plotted by the calculation without evanescent waves, problems concerning accuracy occur at the energy where the number of propagating waves changes, because the number of the waves, which are used to expand the SWFs and affect the accuracy of the SWFs, suddenly increases or decreases. The channel transmission of a graphene sheet with a B-N line defect, which is referred to as Model 1a in the following section, varies smoothly over the energy range that two electronic bands transit from the propagating wave to the evanescent one and the number of propagating waves decreases from three to one. In addition, the error in the sum of the transmission and reflection probabilities, $|1-(R+T)|$, in this energy range is at the level of $10^{-9}$ to $10^{-14}$, which is comparable to the errors in the other energies. Therefore, we conclude that the degradation of the accuracy does not occur in transmission spectra. See Fig. S2 in the Supplemental Material [22] for more details about the error evaluation. (a) Calculation Model 1

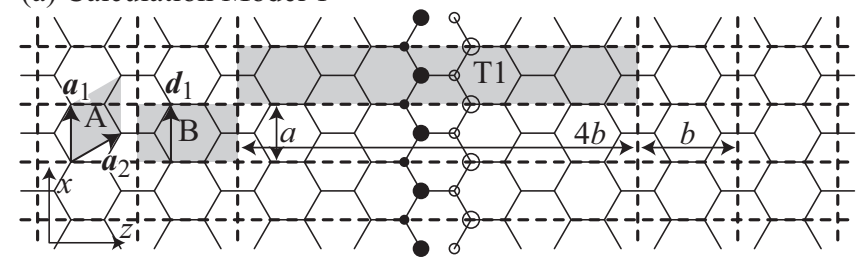

(b) Calculation Model 2

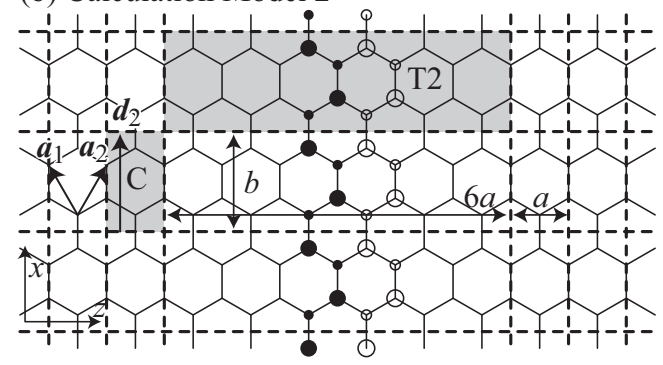

(c) Brillouin zone of unit cell A<smiles>[Y][Y14]1([I-])CC[IH]CC[IH]1</smiles>

(d) Brillouin zone of unit cell B

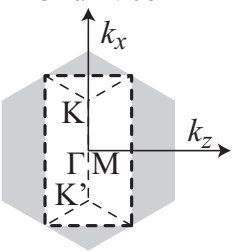

(e) Brillouin zone of unit cell $\mathrm{C}$

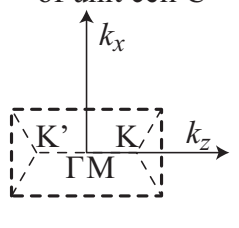

FIG. 5. Schematic representations of graphene sheets with B-N line defects and Brillouin zones of graphene electrodes. In panels (a) and (b), $\mathrm{C}$ atoms are at the corners of the hexagons, and the small solid and open circles denote $\mathrm{B}$ atoms and the large ones $\mathrm{N}$ atoms. The heteroatoms form a zigzag- or armchair-shaped B-N line defect in each graphene sheet. The dashed lines define the unit cells of the transition regions (T1 and T2) and those of the electrode unit cells (B, and C) with $a=2.46 \AA\left(4.65 a_{\mathrm{B}}\right)$ and $b=\sqrt{3} a$, while the rhombus labeled A represents the primitive unit cell of a pristine graphene sheet. The vectors $\boldsymbol{d}_{1}$ and $\boldsymbol{d}_{2}$ denote the translation vectors of the electrode unit cells B and C, respectively. The set of vectors $\left(\boldsymbol{a}_{1}, \boldsymbol{a}_{2}\right)$ is the primitive translation vectors. Panels (c)-(e) illustrate the first Brillouin zones of the electrode unit cells A, B, and C, respectively. The symbols $\Gamma, M, K$, and $\mathrm{K}^{\prime}$ in panel (c) represent the high-symmetry points, and those in panels (d) and (e) represent the equivalent points after folding the hexagonal Brillouin zone.

\section{APPLICATION}

In this section, we present an application of the ballistic electron transport calculation method discussed in the previous sections to two-dimensional materials composed of graphene sheets. Graphene is a well-known two-dimensional (2D) material, and it has been extensively studied for a number of decades [38-41]. Recently, graphene sheets with structural defects, such as pentagon, septagon, octagon rings, and chemical defects, such as substitutional defects with B and/or $\mathrm{N}$ atoms, have attracted a great deal of interest [42-45]. Here, we examine in-plane electron transport of graphene sheets with B-N line defects.

Figures 5(a) and 5(b) show schematic representations of two different calculation models employed in this study. The first model depicted in Fig. 5(a) is referred to as Model 1 hereafter. Model 1 has $\mathrm{C}-\mathrm{C}$ bonds parallel to the $z$ axis and a 
zigzag-shaped B-N line defect along the $x$ direction. On the other hand, the second model, depicted in Fig. 5(b), has C-C bonds perpendicular to the $z$ axis and an armchair-shaped B-N line defect along the $x$ direction. We examine two different widths of the line defects for each model, i.e., one is a single-width line defect as indicated by the solid circles in Figs. 5(a) and 5(b), and the other is a double-width one as indicated by both solid and open circles in Figs. 5(a) and 5(b). The calculation models with the single-width line defect have the suffix "a," i.e., Model 1a and Model 2a, and those with the double-width one the suffix "b," i.e., Model $1 b$ and Model $2 \mathrm{~b}$. Although the primitive unit cell of a graphene sheet used for the electrodes is a rhombus containing two carbon atoms [see rhombus A in Fig. 5(a)], we employ a rectangular unit cell containing four carbon atoms as the unit cell of the graphene electrodes [see rectangle B in Fig. 5(a)]. The transition region of Model 1 is defined as rectangle T1, and the length in the $z$ direction is four times that of the electrode unit cell B so that the B-N line defect is at the middle of T1, as shown in Fig. 5(a). For the other graphene sheet depicted in Fig. 5(b), we define the electrode unit cell as the rectangular four-atom unit cell $\mathrm{C}$ and the transition region as rectangle $\mathrm{T} 2$. The length of $\mathrm{T} 2$ in the $z$ direction is six times that of the electrode unit cell $\mathrm{C}$, so that Model 2 has the B-N line defect at the middle of T2, as shown in Fig. 5(b).

As for the primitive rhombus two-atom unit cell of a graphene sheet, which is indicated by A in Fig. 5(a), it is known that the first Brillouin zone is hexagonal and the high-symmetry $\mathrm{K}$ and $\mathrm{K}^{\prime}$ points appear at the corners of the hexagon as represented in Fig. 5(c), where charge carriers are characterized by a linear dispersion in momentum-energy $(\boldsymbol{k}-E)$ space, and the electronic bands form the Dirac cone around the Fermi energy $E_{F}$. Here, we use rectangular four-atom unit cells B and $\mathrm{C}$ instead of the primitive two-atom unit cells, and thus, the hexagonal Brillouin zone is folded into a rectangular zone, as depicted in Figs. 5(d) and 5(e). As a consequence of the folding, the high-symmetry $\mathrm{K}$ and $\mathrm{K}^{\prime}$ points appear inside the rectangular Brillouin zones, as indicated in Figs. 5(d) and 5(e).

Figure 6 shows the electronic band structure of a graphene sheet around the Fermi energy $E_{\mathrm{F}}$, as calculated with the rectangular four-atom unit cell. The band structure is in good agreement with that calculated by a tight-binding approach with the rectangular four-atom unit cell [46]. The Dirac points are clearly visible at the $\mathrm{K}$ and $\mathrm{K}^{\prime}$ points in the rectangular Brillouin zone. In the energy window between $-1 \mathrm{eV}$ and +1 $\mathrm{eV}$, one can see only the electronic states forming the Dirac cone, meaning that the graphene electrodes have conducting electrons with their momentum around the $\mathrm{K}$ and $\mathrm{K}^{\prime}$ points within the energy window. More specifically, in the case of Model 1, since the Dirac points are at the $\mathrm{K}$ and $\mathrm{K}^{\prime}$ points in Fig. 5(d), i.e., $\left(k_{x}, k_{z}\right)=( \pm 1 / 3,0)$ [47], the electrons conducting through the graphene electrodes and entering T1 have transverse momenta only around $k_{x}= \pm 1 / 3$. In the case of Model 2, the Dirac points are at the $\mathrm{K}$ and $\mathrm{K}^{\prime}$ points in Fig. 5(e), i.e., $\left(k_{x}, k_{z}\right)=(0, \pm 1 / 3)$, and hence, the conducting electrons of the graphene electrodes have transverse momenta only around $k_{x}=0$. Transverse momenta and energies allowed for the electrons conducting through pristine graphene sheets are schematically represented by the gray triangles in Figs. 6(b) and 6(c), referred to as the Dirac triangles hereafter. The

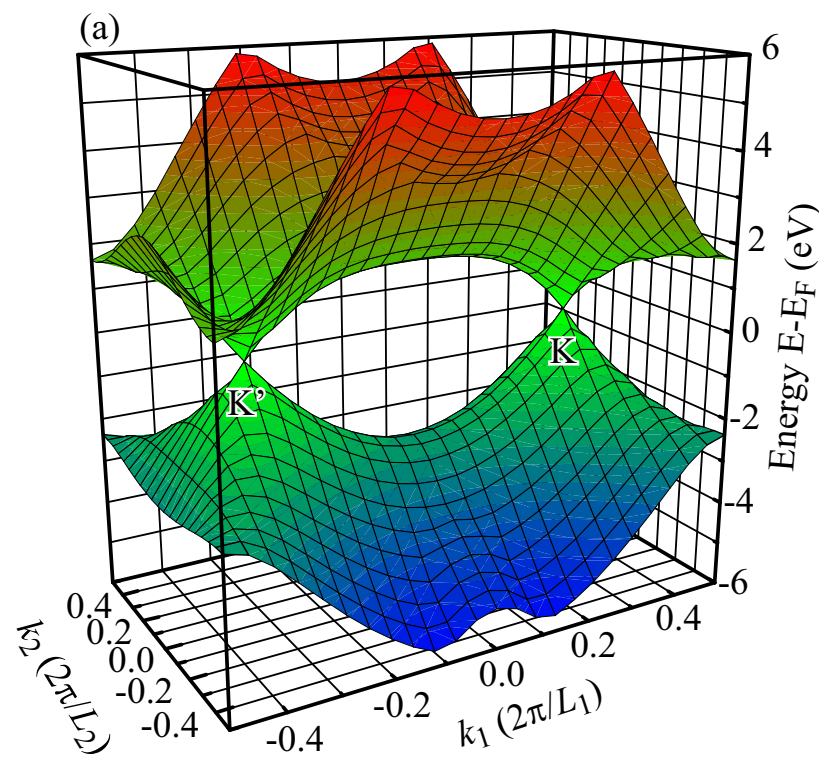

(b)

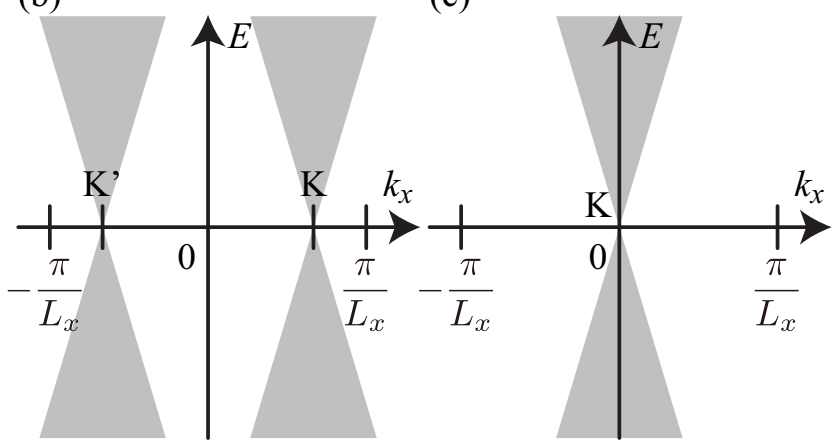

FIG. 6. Electronic band structure of a pristine graphene sheet calculated with the rectangular four-atom unit cell, as illustrated in Figs. 5(a) and 5(b). In panel (a), the axis $k_{1(2)}$ corresponds to the axis $k_{x(y)}$ in Fig. 5(d), and the axis $k_{y(x)}$ in Fig. 5(e). $L_{1}$ and $L_{2}$ denote the lengths of the short and long sides of the rectangular four-atom unit cell, i.e., $L_{1}=a$ and $L_{2}=b=\sqrt{3} a$, respectively. The Dirac points are labeled $\mathrm{K}$ and $\mathrm{K}^{\prime}$. Panels (b) and (c) depict projections of the band structure on the transversal momentum-energy $\left(k_{x}-E\right)$ plane for Model 1 and 2, respectively. The gray areas are referred to as Dirac triangles in the text.

Dirac triangles agree well with the classification of graphene transport behavior by Yazyev and Louie [42]. According to the classification, Model 1 is class $\mathrm{Ib}$, because the translation vector $\boldsymbol{d}_{1}$ [see Fig. 5(a)] has the index $(n, m)=(1,0)$ satisfying $n-m \neq 3 q$. On the other hand, Model 2 is class Ia, because the translation vector $\boldsymbol{d}_{2}$ [see Fig. 5(b)] has the index $(n, m)=(1,1)$ satisfying $n-m=3 q$.

The electron transport calculations of the graphene sheets with the B-N line defects are carried out using the code $[11,12,15,24,25]$ incorporating the aforementioned technique together with the WFM method based on the density functional theory $[31,32,48]$. The generalized Bloch wave functions and scattering wave functions are determined in a non-self-consistent manner to a set of given potential and pseudopotential parameters, which are used for constructing the Kohn-Sham matrix $E \mathbf{S}-\mathbf{H}$ in Eqs. (2) and (B1) for the electrode and transition regions, respectively. In the calculation 
of the generalized Bloch states of the graphene electrodes, we adopt the cutoff parameter $\lambda_{\min }=0.316$. Electron transmission is determined from the scattering wave functions using Eq. (54). The effective potential and pseudopotential parameters are determined in advance under periodic boundary conditions by using the electronic structure calculation code RSPACE [12,33-35], which is based on the real-space finite-difference formalism [36,37], as well as the transport calculation code, in order to treat the physical quantities on the same footing. The interaction between the valence electrons and atomic nuclei is treated through the projector-augmented wave pseudopotential method proposed by Blöchl [49,50], and the exchange-correlation interaction is calculated using the local density approximation proposed by Vosko, Wilk, and Nasair [51], which is within the framework of the density functional theory $[31,32,48]$. In electron transport calculations we assume that incident electron waves are injected from the left electrode.

Before discussing the physics in electron transport through the graphene sheet with a B-N defect, we briefly mention about the error in the sum of the transmission and reflection probabilities. The error obtained by the present method using regular inverses is confirmed to be at the order of $10^{-14}$ to $10^{-10}$, and that obtained by the conventional method using pseudoinverses is of $10^{-3}$ to $10^{-1}$, which is similar to the case of $l=0$ in Fig. 4. See Fig. S3 in the Supplemental Material [22] for the detailed values. Taking into account that it is time consuming to compute a large number of evanescent waves or the SWFs of a transition region with extra layers, we can conclude that the error obtained by the conventional method is too large to discuss the electron transmission and reflection probabilities of the systems considered here.

Let us move to the discussion on the electron transport properties of the graphene sheets. Firstly, we consider electron transport through a pristine graphene sheet without any defects. As one can see from Fig. 5(d), the rectangular Brillouin zone of the graphene electrode for Model 1 has Dirac points on the line $k_{z}=0$, and thus, a half of each Dirac cone belongs to the longitudinal momentum $k_{z}>0$ and the other half $k_{z}<0$. Therefore, only the electrons belonging to the half Dirac cones at one side propagate in the positive $z$ direction, and those belonging to the half Dirac cone at the other side in the negative $z$ direction. This means that there is only one incident wave toward the transition region from an electrode for every transverse momentum $k_{x}$ and energy $E$. Consequently, when T1 is composed only of a pristine graphene sheet without any heteroatoms, the electron transmission is uniform and quantized to 1 over the Dirac triangles on a $k_{x}-E$ plane, as represented in Fig. 6(b). Now we discuss the electron transport through the graphene sheets with single and double zigzag-shaped B-N line defects. The electron transmission of Models $1 \mathrm{a}$ and $1 \mathrm{~b}$ are shown as functions of the transverse momentum $k_{x}$ and the energy $E$ in Figs. 7(a) and 7(b), respectively. One can easily see that the transmission quantization does not appear when a zigzag-shaped B-N line defect is in T1. More specifically, the contour maps show that the electron transmission has a broad and nonquantized peak for the transverse momentum $k_{x}<\mathrm{K}$ and decreases toward the Dirac cone at $K_{x}>\mathrm{K}$. This implies that the dependency of the electron transmission on the transverse momentum $k_{x}$ emerges due to the introduction of $\mathrm{B}-\mathrm{N}$ line defects. Comparing

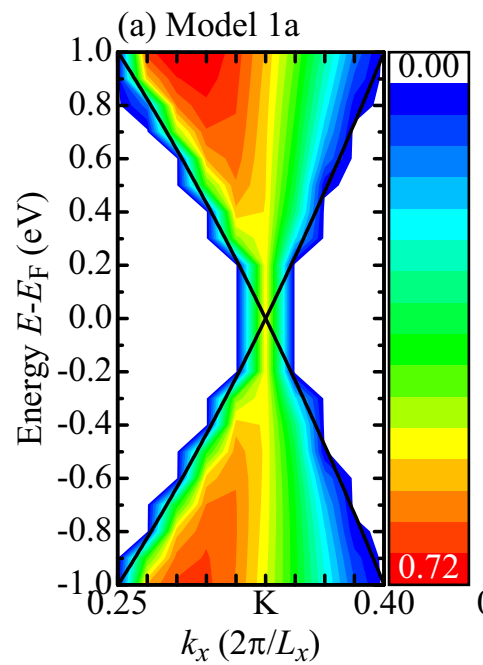

(b) Model 1b

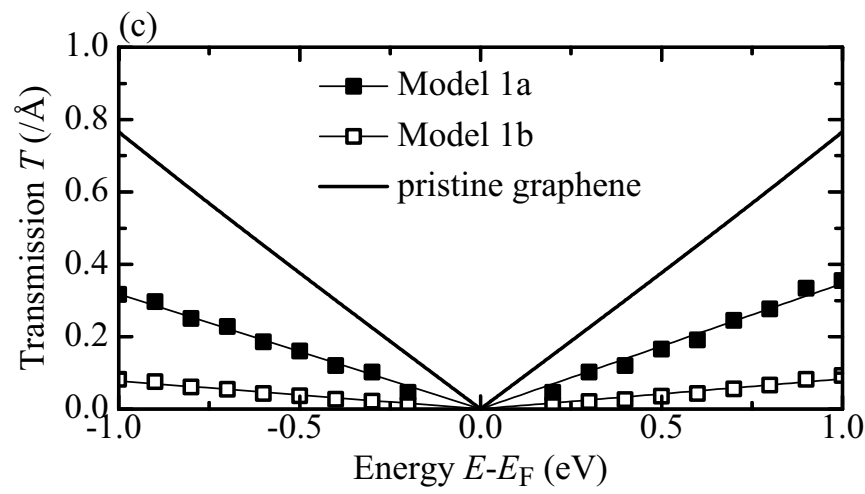

FIG. 7. Electron transmissions of graphene sheets with zigzagshaped B-N line defect [Model 1 in Fig. 5(a)]. In panels (a) and (b), electron transmission contours are plotted as functions of the transverse momentum $k_{x}$ and energy $E$ with respect to the Fermi energy $E_{\mathrm{F}}$ for Model $1 \mathrm{a}$ and $1 \mathrm{~b}$, respectively. The solid curves describe the Dirac bands of a pristine graphene sheet around the Dirac point K. In panel (c), the transmission spectra per unit length $\AA$ for Models $1 \mathrm{a}$ and $1 \mathrm{~b}$ are plotted together with the transmission spectrum of a pristine graphene sheet. The data points indicated by the solid and open squares are evaluated from Eq. (56), and the data points around the Fermi energy $E_{\mathrm{F}}$ are not evaluated because of too few sampling points.

the two electron transmission contours, one can see that only the magnitude of the electron transmission changes and the tendencies of the transmission distributions over the $k_{x}-E$ plane are almost the same, when the width of the B-N line defect is changed.

Figure 7(c) shows the electron transmission per unit length $\AA$ for Model 1a, 1b, and a pristine graphene sheet as a function of the energy of incident electrons. The electron transmission spectrum $T(E)$ is, in general, evaluated by integrating the total electron transmission function $T_{\text {total }}\left(k_{x}, E\right)=\sum_{i} T_{i}\left(k_{x}, E\right)$ over the transverse momentum $k_{x}$, where $T_{i}\left(k_{x}, E\right)$ represents the electron transmission of the $i$ th transmission channel:

$$
\begin{aligned}
T_{\mathrm{BN} @ \mathrm{Gr}}(E) & =\sum_{i} \int_{-\pi / L_{x}}^{+\pi / L_{x}} T_{i}\left(k_{x}, E\right) \mathrm{d} k_{x} \\
& \approx \frac{1}{N} \frac{2 \pi}{L_{x}} \sum_{i, j} T_{i}\left(k_{x, j}, E\right) .
\end{aligned}
$$


$L_{x}$ is the length of the unit cell in the $x$ direction, and $L_{x}=a$ for Model 1. In the practical evaluation, we use Eq. (56) in which $j$ indicates a discrete point in the transverse momentum $k_{x}$. In the case of a pristine graphene sheet, the total electron transmission is known to be uniformly quantized over the Dirac triangles, and therefore, the electron transmission spectrum is expressed in a simple form using the width of the Dirac triangle in the $k_{x}$ direction, $W_{\mathrm{D}}(E)$, as

$$
T_{\mathrm{Gr}}(E)=2 W_{\mathrm{D}}(E) \frac{2 \pi}{L_{x}},
$$

where the factor 2 arises from the fact that two Dirac cones centered at $\mathrm{K}$ and $\mathrm{K}^{\prime}$ have to be considered. One can see from Fig. 7(c) that introducing/widening the B-N line defect drastically decreases the transmission values, though the linearity of the transmission spectra of the pristine graphene sheet is preserved.

In the case of Model 2, the Dirac cones center at $\left(k_{x}, k_{z}\right)=$ $(0, \pm 1 / 3)$ as seen in Fig. 5(e) and overlap each other on a $k_{x}-E$ plane. Therefore, there are four electron waves propagating in the $z$ direction for every transverse momentum $k_{x}$ and energy $E$. Two of them are heading toward the transition region, and the other two are in the opposite direction. Consequently, the electron transmission of a pristine graphene sheet without any heteroatoms is uniform and quantized to 2 over the Dirac triangles on a $k_{x}-E$ plane, as represented in Fig. 6(c). The electron transmission of the graphene sheets with the single and double armchair-shaped B-N line defects, i.e., Models 2a and $2 \mathrm{~b}$, are shown as functions of the transverse momentum $k_{x}$ and the energy $E$ in Figs. 8(a) and 8(b), respectively. Similar to the case of Model 1, the contour maps exhibit that the electron transmission is drastically reduced by the introduction of the armchair-shaped B-N line defects; the electron transmission values especially around $k_{x}=0$ decrease more than those at around the edges of the Dirac triangles. However, the variation in the electron transmission over the Dirac triangles is not as large as in the case of Model 1.

The electron transmission spectra per unit length $\AA$ for Models $2 \mathrm{a}, 2 \mathrm{~b}$, and a pristine graphene sheet are drawn as a function of the energy of incident electrons in Fig. 8(c). The electron transmission spectrum for the pristine graphene sheet is evaluated using Eq. (57) with $L_{x}=b=\sqrt{3} a$; however, the factor 2 in this case means the two propagating waves entering T2 for every transverse momentum $k_{x}$ and energy $E$. The other two transmission spectra for Models $2 \mathrm{a}$ and $2 \mathrm{~b}$ are evaluated from the electron transmission values plotted in Figs. 8(a) and 8(b) using Eq. (56). The electron transmission spectra for Models $2 \mathrm{a}$ and $2 \mathrm{~b}$ are almost linear and there appear to be no transmission peaks and valleys caused by the resonance/antiresonance of incident electron waves with the defect states, as is the case in Models 1a and 1b. Comparing the electron transmission spectra for Models 1 and 2, one can suppose that the graphene sheet with the B-N defects has almost isotropic electron transport properties as does the pristine graphene sheet, because the electron transmission spectra for the graphene sheets with the single (double) zigzag- and armchair-shaped B-N defects agree well with each other.

Now, let us investigate the difference between Models 1 and 2 as regards the variation in the electron transmission

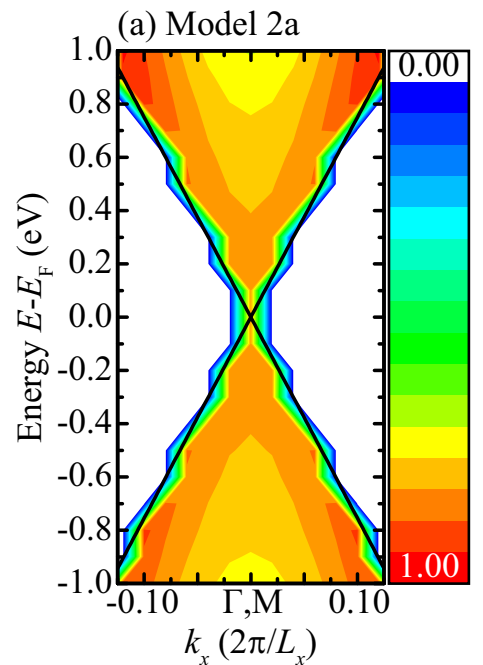

(b) Model 2b

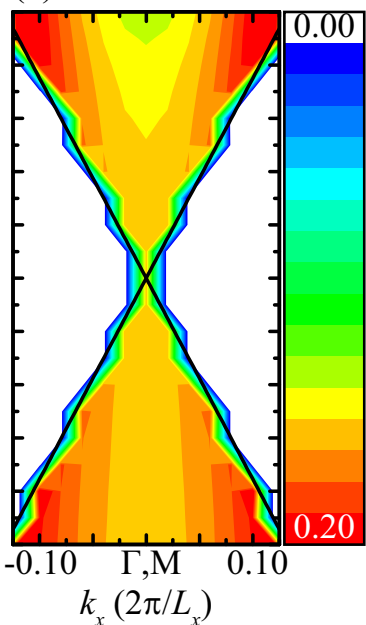

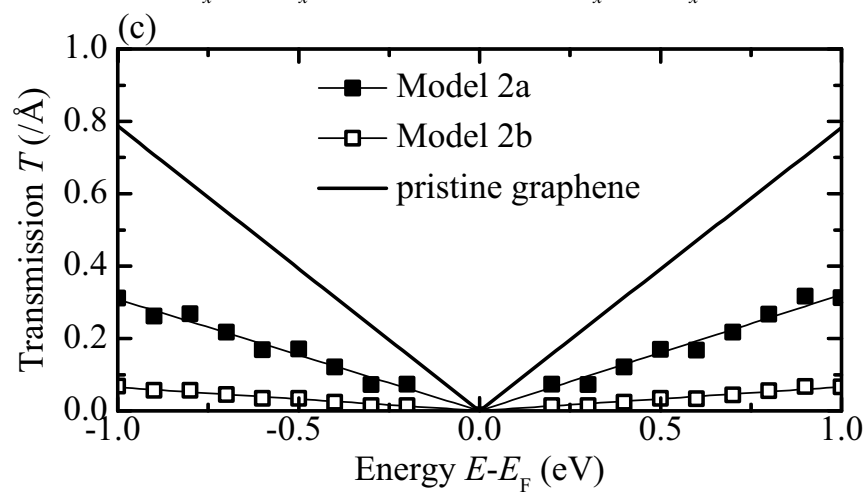

FIG. 8. Electron transmissions of the graphene sheets with armchair-shaped B-N line defect [Model 2 in Fig. 5(b)]. In panels (a) and (b), electron transmission contours are plotted as functions of the transverse momentum $k_{x}$ and energy $E$ with respect to the Fermi energy $E_{\mathrm{F}}$ for Models $2 \mathrm{a}$ and $2 \mathrm{~b}$, respectively. The solid curves describe the Dirac bands of a pristine graphene sheet around the Dirac point K. In panel (c), the transmission spectra per unit length $\AA$ for Models $2 \mathrm{a}$ and $2 \mathrm{~b}$ are plotted together with the transmission spectrum of a pristine graphene sheet. The data points indicated by the solid and open squares are evaluated from Eq. (56), and the data points around the Fermi energy $E_{\mathrm{F}}$ are not evaluated because of too few sampling points.

values inside the Dirac triangles, which have been mentioned together with the electron transmission contours in Figs. 7 and 8. Figures 9(a) and 9(b) illustrate typical electron transmission profiles of Models 1a and 2a extracted from the contour maps in Figs. 7(a) and 8(a), respectively. The electron transmission of Model 1a rapidly increases to $T=0.64$ as the transverse momentum $k_{x}$ increases from 0.25 to 0.3 , while it gradually decreases as the transverse momentum $k_{x}$ changes from 0.3 to 0.4 through the $\mathrm{K}$ point. On the other hand, the electron transmission of Model 2a rapidly increases up to $T=0.92$ around the transverse momentum $k_{x}= \pm 0.1$, and then it gently decreases toward the center $k_{x}=0$. This variation in the electron transmission profile for Model $2 \mathrm{a}$ can also be seen in the channel-decomposed transmission profiles; in particular, the transmission profile for the second channel preserves the original rectangular-shaped electron 


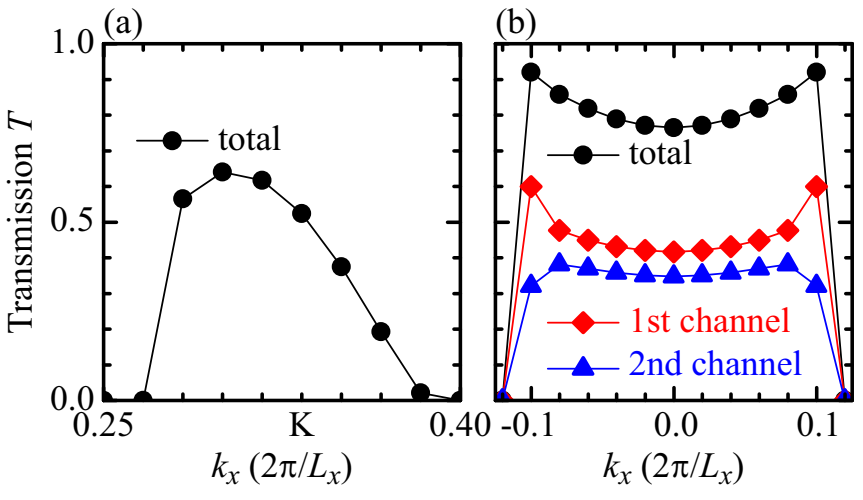

FIG. 9. Typical electron transmission profiles of graphene sheets with single zigzag- and armchair-shaped B-N line defects. The electron transmissions are extracted from Fig. 7(a) and 8(a) for the energy of $-0.8 \mathrm{eV}$. Panel (b) illustrates the first- and second-channel transmission profiles as well as the total one.

transmission profile of the pristine graphene sheet. We can say that the electron transmission of Model 1a depends on the transverse momentum $k_{x}$ more significantly than that of Model 2a.

Let us discuss the electron-transmission dependence on the transverse momentum $k_{x}$ from the viewpoint of the electronic band structures of the transition regions T1 and T2, which are calculated under periodic boundary conditions. Figures 10(a) and $10(\mathrm{~b})$ depict the electronic band structures projected onto $k_{x}-E$ planes for the transition regions $\mathrm{T} 1$ and $\mathrm{T} 2$ of
Models 1a and 2a, respectively. From the electronic band structure of Model 1a, one can easily see that the original band structure of the pristine graphene sheet is largely deformed by the introduction of the zigzag-shaped B-N line defect. This deformation is attributed to hybridization of the Dirac cone states with defect states, because the spatial distribution of the electronic state changes between localized and delocalized as the transverse momentum $k_{x}$ changes as shown in Fig. 10(c). More specifically, the electronic states for the transverse momentums $k_{x}=0.4$ and 0.5 are localized around the defect, while those for $k_{x}=0.2$ and 0.3 are delocalized over the transition region $\mathrm{T} 1$. Note that the spatial distribution of the $i$ th electronic state, $\rho_{i}\left(z, k_{x}\right)$, is evaluated by

$$
\rho_{i}\left(z, k_{x}\right)=\iiint_{\mathrm{BZ}}\left|\psi_{i}\left(x, y, z, k_{x}, k_{z}\right)\right|^{2} \mathrm{~d} k_{z} \mathrm{~d} x \mathrm{~d} y,
$$

where BZ denotes the Brillouin zone and $\psi$ is the KohnSham wave function obtained from the electronic structure calculations under the periodic boundary conditions. Consequently, the influence of the localized defect states on the electron transmission depends on the transverse momentum $k_{x}$ in the case of Model 1a. On the other hand, in the case of Model 2a, there is no drastic deformation of the band structures caused by the introduction of the armchair-shaped B-N line defect. Moreover, in Fig. 10(d), it can be seen that the electronic states are delocalized over the transition region $\mathrm{T} 2$ within the transversal-momentum range from $k_{x}=0$ to $k_{x}=0.1$. These facts imply that the defect states exist far
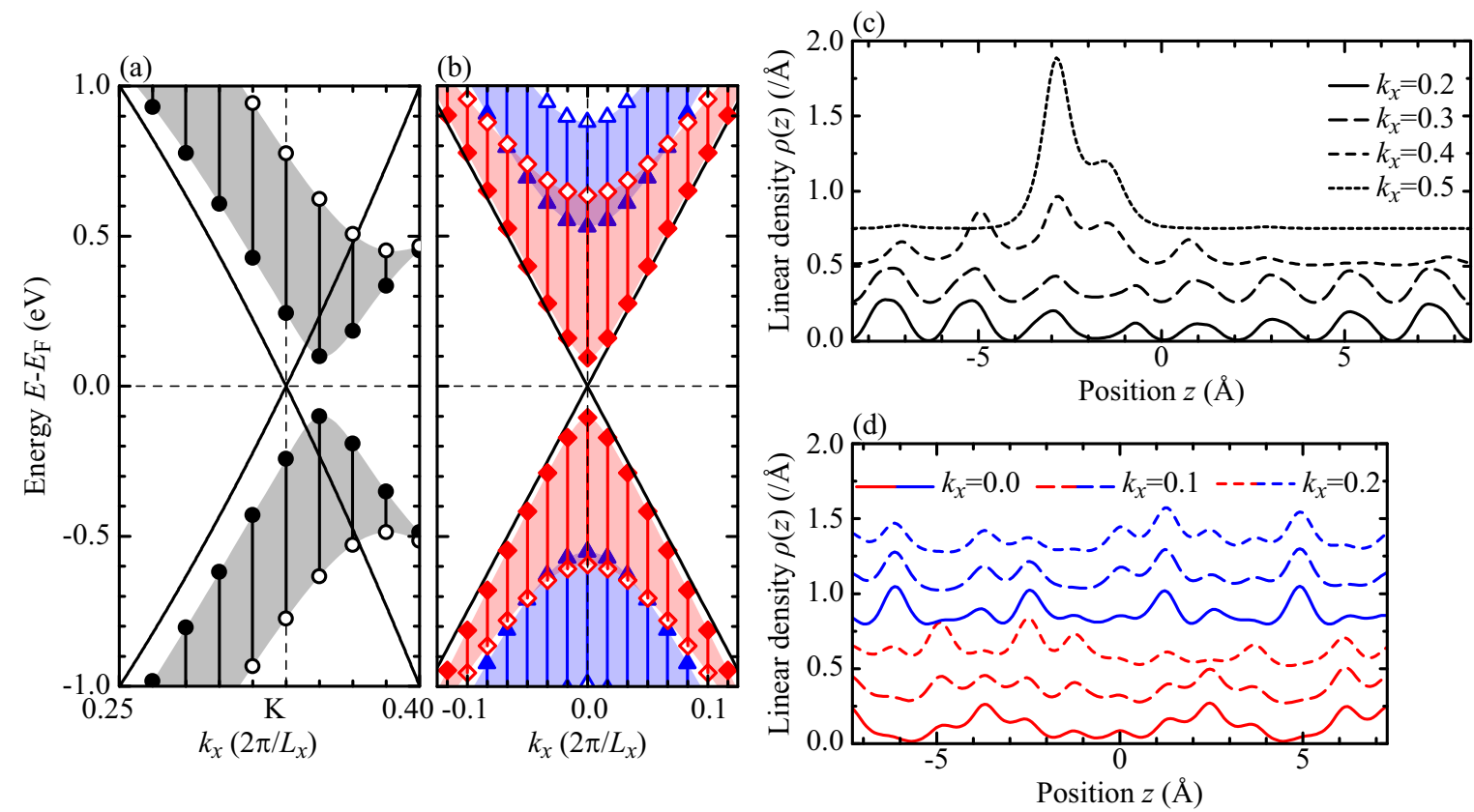

FIG. 10. Electronic band structures and spatial distributions of electrons in the bands. The electronic band structures are calculated for the transition regions T1 and T2 in Fig. 5 under application of periodic boundary conditions. In panels (a) and (b), the electronic band structures as functions of momentums $k_{x}$ and $k_{z}$ are represented as projections onto the $k_{x}-E$ planes for Models 1a and 2a, respectively. The solid and open symbols denote the eigenenergies at $k_{z}=0$ and $k_{z}=0.5$, respectively. The solid curves diagonal to the panels represent the Dirac triangles. Panels (c) and (d) show the spatial distributions of electrons belonging to respective transversal momentum $k_{x}$. In panels (b) and (d), since two electronic bands appear in this energy window, the valence and conduction bands close to the Fermi energy $E_{\mathrm{F}}$ are drawn in red and the others in blue. 
away from the transverse momentum $k_{x}$ and the energy $E$ presented in Fig. 10(b), and hence, the influence on the electron transmission is less dependent on the transverse momentum $k_{x}$. In addition, since no localized defect state exists inside the Dirac triangles shown in Figs. 10(a) and 10(b), resonant electron transport with a quantized transmission value does not occur in Figs. 7(a) and 8(a).

From the electronic band structures in Figs. 10(a) and 10(b), one may expect that the electron transmission is forbidden at certain energy ranges, for instance, $E<-0.8 \mathrm{eV}$ at $k_{x}=\mathrm{K}$ in the case of Model 1a, because of the presence of the band gap. This can be explained by imagining an extension of the supercell $\mathrm{T} 1$ or $\mathrm{T} 2$ in the $z$ direction to separate the interval between the B-N line defects in the neighboring supercells. As the supercell length $L_{z}$ increases, the band dispersion of the delocalized states asymptotically approaches that of a pristine graphene sheet, while the localized defect states form dispersionless flat bands in the longitudinal momentum $k_{z}$ direction. A consequence of this thought experiment is that electrons with energy $E$ and transverse momentum $k_{x}$ within the Dirac triangles are allowed to enter the graphene sheets with B-N line defects sandwiched between the semi-infinite graphene electrodes and to pass through them with a certain transmission probability, which depends on the influence of the localized defect states, as discussed in the previous paragraph.

\section{CONCLUSION}

We reformulated the WFM method for first-principles transport-property calculations so as to exclude the rapidly decreasing evanescent waves because the original WFM methods are formulated to include all the generalized Bloch waves. Sørensen et al. had reported that the transmission probability varies when increasing the number of extra layers attached to the transition region, and the errors in the transmission and reflection probabilities are inconsistent to each other. These results indicate that the translational invariance of the transmission probability with respect to moving the matching planes is not preserved and the sum of these probabilities is not equal to the number of channels in their WFM method. We found that the translational invariance deteriorated as a result of the overlap of the layers between the electrode and transition regions and the exclusion of the rapidly decreasing evanescent waves. Due to the incomplete set of the generalized Bloch waves, pseudoinverses were used to calculate the transmission and reflection probabilities in the conventional WFM methods and caused degradation of the accuracy. We proposed a method that eliminates the overlap of the layers between the transition and electrode regions and computes the transmission probability from the SWFs without the pseudoinverses and the Green's function of the transition region. The proposed method nicely recovers the translational invariance of the transmission probability with respect to insertion of the extra layers and gives transmission and reflection probabilities whose sum exactly agrees with the number of channels. We also demonstrated that the accuracy of the transmission probability of the WFM method without computing the rapidly varying evanescent waves or attaching the extra layers to the transition region is comparable to that obtained by the nonequilibrium Green's function method.
We also carried out electron transport calculations on twodimensional graphene sheets with either zigzag- or armchairshaped B-N line defects and discussed about the indirect influence of the defect states on the electron transport properties. The transport calculations revealed that the graphene sheet with the zigzag-shaped B-N line defect has an electron transmission dependent on transverse momentum perpendicular to the direction of electron transport. On the other hand, the electron transmission of the graphene sheet with the armchair-shaped B-N line defect exhibits less dependency on the transverse momentum, though the transmission value is reduced in comparison to that of ideal electron transport without any defects. The electron-transmission dependency on transverse momentum can be explained from the electronic band structures of the transition regions calculated under periodic boundary conditions. More specifically, the original band structure of a pristine graphene sheet is largely deformed by hybridization with localized states originating from the zigzag-shaped B-N line defect. Because the localized states are at the transverse momentum $k_{x}=0.5$, the incident electrons with transverse momenta close to $k_{x}=0.5$ are significantly affected and the transmission value becomes small. In the case of the armchair-shaped B-N line defect, the band structure is not significantly deformed, because there are no localized states originating from the B-N line defect at the transverse momentum and energy close to the Dirac points. The present study on the graphene sheets using the proposed method can be extended to the study on the electron transport properties of carbon nanotubes with B-N defects, which recently attracts attention [52-54].

\section{ACKNOWLEDGMENTS}

S.T. and S.B. acknowledge the financial support from Deutsche Forschungsgemeinschaft through the Collaborative Research Center SFB 1238 (Project C01). T.O. acknowledges the financial support from MEXT as a social and scientific priority issue (creation of new functional devices and highperformance materials to support next-generation industries) to be tackled by using post-K computer and JSPS KAKENHI Grant No. JP16H03865. The authors would like to thank Professor Kikuji Hirose of Osaka University and Mr. Shigeru Iwase of University of Tsukuba for contributing to our discussions. The numerical calculations were carried out using the supercomputer JURECA at Jülich Supercomputing Centre, Forschungszentrum Jülich, the system B of the Institute for Solid State Physics at the University of Tokyo, the COMA of the Center for Computational Sciences at University of Tsukuba, and the $\mathrm{K}$ computer provided by the RIKEN Advanced Institute for Computational Science through the HPCI System Research project (Project ID: hp160228).

\section{APPENDIX A: ALTERNATIVE APPROACH TO COMPUTE GENERALIZED BLOCH WAVES OF ELECTRODES}

The alternative approach for computation of generalized Bloch waves is to use the Green's function of the isolated Hamiltonian of the electrode $\boldsymbol{\Theta}_{L}\left[\equiv\left(\overline{\mathbf{H}}_{L}\right)^{-1}\right]$ proposed by Fujimoto and Hirose [11]. In this scheme, one assumes that $m_{L}>2 m_{L L}$. If $m_{L}<2 m_{L L}$, several unit cells of the crystal 
are included in $\overline{\mathbf{H}}_{L}$ to satisfy $m_{L}>2 m_{L L}$. The generalized Bloch waves are obtained by solving the following generalized eigenvalue problem

$$
\begin{aligned}
& \left(\begin{array}{cc}
-\boldsymbol{\theta}_{L}\left(\xi_{3}, \xi_{1}\right) \overline{\mathbf{h}}^{L \dagger} & -\boldsymbol{\theta}_{L}\left(\xi_{3}, \xi_{3}\right) \overline{\mathbf{h}}^{L} \\
\mathbf{0} & \mathbf{I}
\end{array}\right)\left(\begin{array}{l}
\phi_{(i-1)}\left(\xi_{3}\right) \\
\phi_{(i+1)}\left(\xi_{1}\right)
\end{array}\right) \\
& =\lambda\left(\begin{array}{cc}
\mathbf{I} & \mathbf{0} \\
-\boldsymbol{\theta}_{L}\left(\xi_{1}, \xi_{1}\right) \overline{\mathbf{h}}^{L \dagger} & -\boldsymbol{\theta}_{L}\left(\xi_{1}, \xi_{3}\right) \overline{\mathbf{h}}^{L}
\end{array}\right)\left(\begin{array}{l}
\phi_{(i-1)}\left(\xi_{3}\right) \\
\phi_{(i+1)}\left(\xi_{1}\right)
\end{array}\right),
\end{aligned}
$$

where

$$
\boldsymbol{\Theta}_{L} \equiv\left(\overline{\mathbf{H}}_{L}\right)^{-1}=\left(\begin{array}{lll}
\boldsymbol{\theta}_{L}\left(\xi_{1}, \xi_{1}\right) & \boldsymbol{\theta}_{L}\left(\xi_{1}, \xi_{2}\right) & \boldsymbol{\theta}_{L}\left(\xi_{1}, \xi_{3}\right) \\
\boldsymbol{\theta}_{L}\left(\xi_{2}, \xi_{1}\right) & \boldsymbol{\theta}_{L}\left(\xi_{2}, \xi_{2}\right) & \boldsymbol{\theta}_{L}\left(\xi_{2}, \xi_{3}\right) \\
\boldsymbol{\theta}_{L}\left(\xi_{3}, \xi_{1}\right) & \boldsymbol{\theta}_{L}\left(\xi_{3}, \xi_{2}\right) & \boldsymbol{\theta}_{L}\left(\xi_{3}, \xi_{3}\right)
\end{array}\right)
$$

Here, multiple grids or bases are bunched together in $\xi_{i} . \xi_{1}$ left (right) neighboring unit cell $\left\{\chi_{1, j}\right\}\left(\left\{\chi_{3}, j\right\}\right)$ and $\xi_{2}$ gathers $m_{L}-2 m_{L L}$ grids or bases not having the interaction with the neighboring cells $\left\{\chi_{2, j}\right\}$. For example,

$$
\xi_{1}=\left(\chi_{1,1}, \ldots, \chi_{1, m_{L L}}\right) .
$$

Thus, $\boldsymbol{\theta}_{L}\left(\xi_{1}, \xi_{1}\right)$ is an $m_{L L} \times m_{L L}$ matrix expressed as $\boldsymbol{\theta}_{L}\left(\xi_{1}, \xi_{1}\right)=\left\langle\xi_{1}\left|\boldsymbol{\Theta}_{L}\right| \xi_{1}\right\rangle$. The computational cost of this scheme is $O\left(m_{L}^{2} m_{L L}\right)$ to set up Eq. (A1), when $\overline{\mathbf{H}}_{L}$ is sparse and an iterative method is used. Although this scheme computes $\left(\xi_{3}\right)$ includes $m_{L L}$ grids or bases having interactions with the

the eigenpairs of Eq. (A1) within the specific interval of $\lambda_{\text {min }}<|\lambda|<1 / \lambda_{\text {min }}$, truly semi-infinite systems can be treated by solving the continued-fraction equations introduced in Sec. IIE.

\section{APPENDIX B: WAVE FUNCTION MATCHING FORMULA}

Let us introduce the WFM formula [11] for the case that the rank of $\overline{\mathbf{H}}_{L}\left(\overline{\mathbf{H}}_{R}\right), m_{L}\left(m_{R}\right)$, and the rank of $\overline{\mathbf{H}}_{L, L}\left(\overline{\mathbf{H}}_{R, R}\right)$, $m_{L L}\left(m_{R R}\right)$, are equal. The case that they are not equal is formulated in Appendix C. The Kohn-Sham equation for the computational model with $l$ extra layers [Fig. 1(b)] is expressed as

$$
\left(E \widehat{\mathbf{S}}^{(l)}-\widehat{\mathbf{H}}^{(l)}\right)\left(\begin{array}{c}
\psi_{1}^{(l)} \\
\vdots \\
\vdots \\
\vdots \\
\psi_{n}^{(l)}
\end{array}\right)=\left(\begin{array}{c}
-\overline{\mathbf{H}}_{L, L}^{\dagger} \psi_{0} \\
0 \\
\vdots \\
0 \\
-\overline{\mathbf{H}}_{R, R} \psi_{n+1}
\end{array}\right)
$$

Multiplying the Green's function $\widehat{\mathbf{G}}^{(l)}\left[=\left(E \widehat{\mathbf{S}}^{(l)}-\widehat{\mathbf{H}}^{(l)}\right)^{-1}\right]$ from the left hand side yields

$$
\left(\begin{array}{c}
\psi_{1}^{(l)} \\
\vdots \\
\vdots \\
\vdots \\
\psi_{n}^{(l)}
\end{array}\right)=\widehat{\mathbf{G}}^{(l)}\left(\begin{array}{c}
-\overline{\mathbf{H}}_{L, L}^{\dagger} \psi_{0} \\
0 \\
\vdots \\
0 \\
-\overline{\mathbf{H}}_{R, R} \psi_{n+1}
\end{array}\right),
$$

where

$$
\widehat{\mathbf{G}}^{(l)}=\left(\begin{array}{ccccccccc}
\mathbf{G}_{1-l, 1-l}^{(l)} & \ldots & \mathbf{G}_{1-l, 0}^{(l)} & \mathbf{G}_{1-l, 1}^{(l)} & \ldots & \mathbf{G}_{1-l, n}^{(l)} & \mathbf{G}_{1-l, n+1}^{(l)} & \ldots & \mathbf{G}_{1-l, n+l}^{(l)} \\
\vdots & \ddots & \vdots & \vdots & & \vdots & \vdots & & \vdots \\
\mathbf{G}_{0,1-l}^{(l)} & \ldots & \mathbf{G}_{0,0}^{(l)} & \mathbf{G}_{0,1}^{(l)} & \ldots & \mathbf{G}_{0, n}^{(l)} & \mathbf{G}_{0, n+1}^{(l)} & \ldots & \mathbf{G}_{0, n+l}^{(l)} \\
\mathbf{G}_{1,1-l}^{(l)} & \ldots & \mathbf{G}_{1,0}^{(l)} & \mathbf{G}_{1,1}^{(l)} & \ldots & \mathbf{G}_{1, n}^{(l)} & \mathbf{G}_{1, n+1}^{(l)} & \ldots & \mathbf{G}_{1, n+l}^{(l)} \\
\vdots & & \vdots & \vdots & \ddots & \vdots & \vdots & & \vdots \\
\mathbf{G}_{n, 1-l}^{(l)} & \ldots & \mathbf{G}_{n, 0}^{(l)} & \mathbf{G}_{n, 1}^{(l)} & \ldots & \mathbf{G}_{n, n}^{(l)} & \mathbf{G}_{n, n+1}^{(l)} & \ldots & \mathbf{G}_{n, n+l}^{(l)} \\
\mathbf{G}_{n+1,1-l}^{(l)} & \ldots & \mathbf{G}_{n+1,0}^{(l)} & \mathbf{G}_{n+1,1}^{(l)} & \ldots & \mathbf{G}_{n+1, n}^{(l)} & \mathbf{G}_{n+1, n+1}^{(l)} & \ldots & \mathbf{G}_{n+1, n+l}^{(l)} \\
\vdots & & \vdots & \vdots & & \vdots & \vdots & \ddots & \vdots \\
\mathbf{G}_{n+l, 1-l}^{(l)} & \ldots & \mathbf{G}_{n+l, 0}^{(l)} & \mathbf{G}_{n+l, 1}^{(l)} & \ldots & \mathbf{G}_{n+l, n}^{(l)} & \mathbf{G}_{n+l, n+1}^{(l)} & \ldots & \mathbf{G}_{n+l, n+l}^{(l)}
\end{array}\right)
$$

with $\mathbf{G}_{i, j}^{(l)}$ being the $(i, j)$ th block-matrix element of the Green's function of the isolated transition region $\widehat{\mathbf{G}}^{(l)}$. From the first and last block rows of Eq. (B2), $\psi_{0}$ and $\psi_{n+1}$ are related to $\psi_{1}^{(l)}$ and $\psi_{n}^{(l)}$ as follows:

$$
\begin{aligned}
\psi_{1}^{(l)} & =-\mathbf{G}_{1-l, 1-l}^{(l)} \overline{\mathbf{H}}_{L, L}^{\dagger} \psi_{0}-\mathbf{G}_{1-l, n+l}^{(l)} \overline{\mathbf{H}}_{R, R} \psi_{n+1}, \\
\psi_{n}^{(l)} & =-\mathbf{G}_{n+l, 1-l}^{(l)} \overline{\mathbf{H}}_{L, L}^{\dagger} \psi_{0}-\mathbf{G}_{n+l, n+l}^{(l)} \overline{\mathbf{H}}_{R, R} \psi_{n+1} .
\end{aligned}
$$

Substituting the relation of Eq. (8) into Eqs. (B4) and (B5), we obtain

$$
\begin{gathered}
\psi_{1}^{\mathrm{ref},(l)}+\psi_{1}^{\mathrm{in},(l)}=-\mathbf{G}_{1-l, 1-l}^{(l)} \overline{\mathbf{H}}_{L, L}^{\dagger}\left(\psi_{0}^{\mathrm{ref}}+\psi_{0}^{\mathrm{in}}\right)-\mathbf{G}_{1-l, n+l}^{(l)} \overline{\mathbf{H}}_{R, R} \psi_{n+1}, \\
\psi_{n}^{(l)}=-\mathbf{G}_{n+l, 1-l}^{(l)} \overline{\mathbf{H}}_{L, L}^{\dagger}\left(\psi_{0}^{\mathrm{ref}}+\psi_{0}^{\mathrm{in}}\right)-\mathbf{G}_{n+l, n+l}^{(l)} \overline{\mathbf{H}}_{R, R} \psi_{n+1} .
\end{gathered}
$$


Rewriting Eqs. (B6) and (B7) in terms of the SWFs in the electrode regions $\psi_{0}^{\text {ref }}$ and $\psi_{n+1}$, we arrive at the following WFM formula,

$$
\left(\begin{array}{cc}
-\mathbf{G}_{1-l, 1-l}^{(l)} \overline{\mathbf{H}}_{L, L}^{\dagger}-\psi_{1}^{\mathrm{ref},(l)}\left(\psi_{0}^{\mathrm{ref}}\right)^{-1} & -\mathbf{G}_{1-l, n+l}^{(l)} \overline{\mathbf{H}}_{R, R} \\
-\mathbf{G}_{n+l, 1-l}^{(l)} \overline{\mathbf{H}}_{L, L}^{\dagger} & -\mathbf{G}_{n+l, n+l}^{(l)} \overline{\mathbf{H}}_{R, R}-\psi_{n}^{(l)}\left(\psi_{n+1}\right)^{-1}
\end{array}\right)\left(\begin{array}{c}
\psi_{0}^{\text {ref }} \\
\psi_{n+1}
\end{array}\right)=\left(\begin{array}{c}
\mathbf{G}_{1-l, 1-l}^{(l)} \overline{\mathbf{H}}_{L, L}^{\dagger} \psi_{0}^{\text {in }}+\psi_{1}^{\text {in, },(l)} \\
\mathbf{G}_{n+l, 1-l}^{(l)} \overline{\mathbf{H}}_{L, L}^{\dagger} \psi_{0}^{\text {in }}
\end{array}\right) .
$$

Here, $\psi_{0}^{\text {in }}$ is determined by Eq. (23) and $\psi_{1}^{\text {in, }(l)}=\lambda_{L, k}^{+} \psi_{0}^{\text {in }}$. In Eq. (B8), the left (right) matching plane is set between $\psi_{0}^{\text {ref }}$ and $\psi_{1}^{\text {ref, },(l)}\left(\psi_{n}^{(l)}\right.$ and $\left.\psi_{n+1}\right)$.

To solve the simultaneous equations of Eq. (B8), we need the ratios of the SWFs between the neighboring layers on both sides of the matching planes $\psi_{1}^{\text {ref, }(l)}\left(\psi_{0}^{\text {ref }}\right)^{-1}$ and $\psi_{n}^{(l)}\left(\psi_{n+1}\right)^{-1}$. From the boundary conditions in the electrode regions, i.e., Eqs. (22)-(25), the ratios in the right and left electrode regions are given by

$$
\psi_{n+1}\left(\psi_{n+2}\right)^{-1}=\widetilde{\boldsymbol{\Phi}}_{R}^{+}\left(\widetilde{\boldsymbol{\Phi}}_{R}^{+} \widetilde{\mathbf{\Lambda}}_{R}^{+}\right)^{-1},
$$

and

$$
\psi_{0}^{\mathrm{ref}}\left(\psi_{-1}^{\mathrm{ref}}\right)^{-1}=\widetilde{\boldsymbol{\Phi}}_{L}^{-}\left(\widetilde{\boldsymbol{\Phi}}_{L}^{-}\left(\widetilde{\mathbf{\Lambda}}_{L}^{-}\right)^{-1}\right)^{-1},
$$

respectively. Here, $\widetilde{\boldsymbol{\Phi}}_{R}^{+}$and $\widetilde{\boldsymbol{\Phi}}_{L}^{-}$contain only the propagating and slowly varying evanescent waves. Thus, $\left(\widetilde{\boldsymbol{\Phi}}_{R}^{+} \widetilde{\boldsymbol{\Lambda}}_{R}^{+}\right)^{-1}$ and $\left(\widetilde{\boldsymbol{\Phi}}_{L}^{-}\left(\widetilde{\boldsymbol{\Lambda}}_{L}^{-}\right)^{-1}\right)^{-1}$ are determined by the pseudoinverses. In the left electrode region, the SWF satisfies

$$
\overline{\mathbf{H}}_{L, L}^{\dagger} \psi_{-1}^{\mathrm{ref}}+\overline{\mathbf{H}}_{L} \psi_{0}^{\mathrm{ref}}+\overline{\mathbf{H}}_{L, L} \psi_{1}^{\mathrm{ref},(l)}=0
$$

Accordingly, we can see that the ratio of the SWFs at the left matching plane $\psi_{1}^{\text {ref, }(l)}\left(\psi_{0}^{\text {ref }}\right)^{-1}$ can be derived as

$$
\psi_{1}^{\text {ref, }(l)}\left(\psi_{0}^{\text {ref }}\right)^{-1}=-\left(\overline{\mathbf{H}}_{L, L}\right)^{-1}\left(\overline{\mathbf{H}}_{L}+\overline{\mathbf{H}}_{L, L}^{\dagger} \psi_{-1}^{\text {ref }}\left(\psi_{0}^{\text {ref }}\right)^{-1}\right)
$$

In a similar way, the ratio at the right matching plane can be constructed as

$$
\psi_{n}^{(l)}\left(\psi_{n+1}\right)^{-1}=-\left(\overline{\mathbf{H}}_{R, R}^{\dagger}\right)^{-1}\left(\overline{\mathbf{H}}_{R}+\overline{\mathbf{H}}_{R, R} \psi_{n+2}\left(\psi_{n+1}\right)^{-1}\right)
$$

Note that once the recursive equations Eqs. (B12) and (B13) are solved, $\psi_{1}^{\text {ref, },(l)}\left(\psi_{0}^{\text {ref }}\right)^{-1}$ and $\psi_{n}^{(l)}\left(\psi_{n+1}\right)^{-1}$ contain part of the contribution of the rapidly varying evanescent wave excluded by the cutoff parameter $\lambda_{\min }$. Inserting Eqs. (B12) and (B13) into Eq. (B8) and solving the simultaneous equation Eq. (B8), we obtain the SWFs at the first layers of the left and right electrode regions $\psi_{0}^{\text {ref }}$ and $\psi_{n+1}$. 
Here, we define the $m_{L L} \times m_{L L}$ ratio matrices [11] as

$$
\begin{aligned}
& \mathbf{R}^{\mathrm{ref},(l+1)}=\psi_{-1}^{\mathrm{ref}}\left(\psi_{0}^{\mathrm{ref}}\right)^{-1}, \\
& \mathbf{R}^{\mathrm{ref},(l)}=\psi_{0}^{\mathrm{ref}}\left(\psi_{1}^{\mathrm{ref},(l)}\right)^{-1},
\end{aligned}
$$

and the $m_{R R} \times m_{R R}$ ratio matrices as

$$
\begin{aligned}
& \mathbf{R}^{\operatorname{tra},(l+2)}=\psi_{n+2}\left(\psi_{n+1}\right)^{-1}, \\
& \mathbf{R}^{\operatorname{tra},(l+1)}=\psi_{n+1}\left(\psi_{n}^{(l)}\right)^{-1} .
\end{aligned}
$$

By substituting Eqs. (B14) and (B15) [Eqs. (B16) and (B17)] into Eq. (B12) [Eq. (B13)] and shifting the indices of ratio matrices by one unit cell, Eqs. (B12) and (B13) can be rewritten in the form of continued fractions [11],

$$
\begin{aligned}
& \mathbf{R}^{\text {ref, }(l-1)}=-\left(\overline{\mathbf{H}}_{L}+\overline{\mathbf{H}}_{L, L}^{\dagger} \mathbf{R}^{\mathrm{ref},(l)}\right)^{-1} \overline{\mathbf{H}}_{L, L}, \\
& \mathbf{R}^{\text {tra, }(l)}=-\left(\overline{\mathbf{H}}_{R}+\overline{\mathbf{H}}_{R, R} \mathbf{R}^{\text {tra, },(l+1)}\right)^{-1} \overline{\mathbf{H}}_{R, R}^{\dagger} .
\end{aligned}
$$

Accordingly, the WFM formula Eq. (B8) becomes

$$
\left(\begin{array}{cc}
-\mathbf{G}_{1-l, 1-l}^{(l)} \overline{\mathbf{H}}_{L, L}^{\dagger}-\left(\mathbf{R}^{\mathrm{ref},(l)}\right)^{-1} & -\mathbf{G}_{1-l, n+l}^{(l)} \overline{\mathbf{H}}_{R, R} \\
-\mathbf{G}_{n+l, 1-l}^{(l)} \overline{\mathbf{H}}_{L, L}^{\dagger} & -\mathbf{G}_{n+l, n+l}^{(l)} \overline{\mathbf{H}}_{R, R}-\left(\mathbf{R}^{\text {tra, },(l+1)}\right)^{-1}
\end{array}\right)\left(\begin{array}{c}
\psi_{0}^{\mathrm{ref}} \\
\psi_{n+1}
\end{array}\right)=\left(\begin{array}{c}
\mathbf{G}_{1-l, 1-l}^{(l)} \overline{\mathbf{H}}_{L, L}^{\dagger} \psi_{0}^{\text {in }}+\psi_{1}^{\text {in },(l)} \\
\mathbf{G}_{n+l, 1-l}^{(l)} \overline{\mathbf{H}}_{L, L}^{\dagger} \psi_{0}^{\text {in }}
\end{array}\right) .
$$

The SWFs in the transition region are obtained by substituting the obtained $\psi_{0}^{\text {ref }}$ and $\psi_{n+1}$ into Eq. (B2). Note that only parts of the block-matrix elements of the Green's functions matrix $\mathbf{G}_{i, 1-l}^{(l)}$ and $\mathbf{G}_{i, n+l}^{(l)}(i=1-l, \ldots, n+l)$ are needed to obtain the SWFs.

\section{APPENDIX C: WAVE FUNCTION MATCHING FOR GENERAL CASE}

In Appendix B, we introduced the WFM formula [11] for the case that the ranks of $\overline{\mathbf{H}}_{L}\left(\overline{\mathbf{H}}_{R}\right)$ and $\overline{\mathbf{H}}_{L, L}\left(\overline{\mathbf{H}}_{R, R}\right)$ are identical $\left(m_{L}=m_{L L}\right.$ and $\left.m_{R}=m_{R R}\right)$. In many cases, e.g., real-space grid methods, the Hamiltonian does not satisfy such a condition. In this Appendix, the WFM formula for the case that $m_{L}>m_{L L}$ and $m_{R}>m_{R R}$ will be derived. Although we assume $m_{L}>2 m_{L L}$, this condition can be easily satisfied by increasing the number of unit cells in $\overline{\mathbf{H}}_{L}$, as introduced in Sec. II A. The splitting using $\xi_{1}, \xi_{2}$, and $\xi_{3}$ in Sec. II A allows the SWFs $\psi_{1}^{\text {ref, }(l)}$ to be written as

$$
\psi_{1}^{\mathrm{ref},(l)}=\left(\begin{array}{l}
\psi_{1}^{\mathrm{ref},(l)}\left(\xi_{1}\right) \\
\psi_{1}^{\mathrm{ref},(l)}\left(\xi_{2}\right) \\
\psi_{1}^{\mathrm{ref},(l)}\left(\xi_{3}\right)
\end{array}\right) .
$$

Using the same separation for $\psi_{0}, \psi_{n}^{(l)}$, and $\psi_{n+1}$, Eq. (B1) is expressed as

$$
\left(E \widehat{\mathbf{S}}^{(l)}-\widehat{\mathbf{H}}^{(l)}\right)\left(\begin{array}{c}
\psi_{1}^{\mathrm{ref},(l)}\left(\xi_{1}\right) \\
\psi_{1}^{\mathrm{ref},(l)}\left(\xi_{2}\right) \\
\psi_{1}^{\mathrm{ref},(l)}\left(\xi_{3}\right) \\
\vdots \\
\psi_{n}^{(l)}\left(\xi_{1}\right) \\
\psi_{n}^{(l)}\left(\xi_{2}\right) \\
\psi_{n}^{(l)}\left(\xi_{3}\right)
\end{array}\right)=\left(\begin{array}{c}
-\overline{\mathbf{h}}^{L \dagger} \psi_{0}\left(\xi_{3}\right) \\
0 \\
\vdots \\
0 \\
-\overline{\mathbf{h}}^{R} \psi_{n+1}\left(\xi_{1}\right)
\end{array}\right) .
$$


Multiplying $\widehat{\mathbf{G}}^{(l)}\left[=\left(E \widehat{\mathbf{S}}^{(l)}-\widehat{\mathbf{H}}^{(l)}\right)^{-1}\right]$ from the left hand side leads

$$
\left(\begin{array}{c}
\psi_{1}^{\mathrm{ref},(l)}\left(\xi_{1}\right) \\
\psi_{1}^{\mathrm{ref},(l)}\left(\xi_{2}\right) \\
\psi_{1}^{\mathrm{ref},(l)}\left(\xi_{3}\right) \\
\vdots \\
\psi_{n}^{(l)}\left(\xi_{1}\right) \\
\psi_{n}^{(l)}\left(\xi_{2}\right) \\
\psi_{n}^{(l)}\left(\xi_{3}\right)
\end{array}\right)=\widehat{\mathbf{G}}^{(l)}\left(\begin{array}{c}
-\overline{\mathbf{h}}^{L \dagger} \psi_{0}\left(\xi_{3}\right) \\
0 \\
\vdots \\
0 \\
-\overline{\mathbf{h}}^{R} \psi_{n+1}\left(\xi_{1}\right)
\end{array}\right) .
$$

Supposing that $(i, j)$ block-matrix element of Green's function $\mathbf{G}_{i, j}^{(l)}$ in Eq. (B3) is given by

$$
\mathbf{G}_{i, j}^{(l)}=\left(\begin{array}{lll}
\mathbf{G}_{i, j}^{(l)}\left(\xi_{1}, \xi_{1}\right) & \mathbf{G}_{i, j}^{(l)}\left(\xi_{1}, \xi_{2}\right) & \mathbf{G}_{i, j}^{(l)}\left(\xi_{1}, \xi_{3}\right) \\
\mathbf{G}_{i, j}^{(l)}\left(\xi_{2}, \xi_{1}\right) & \mathbf{G}_{i, j}^{(l)}\left(\xi_{2}, \xi_{2}\right) & \mathbf{G}_{i, j}^{(l)}\left(\xi_{2}, \xi_{3}\right) \\
\mathbf{G}_{i, j}^{(l)}\left(\xi_{3}, \xi_{1}\right) & \mathbf{G}_{i, j}^{(l)}\left(\xi_{3}, \xi_{2}\right) & \mathbf{G}_{i, j}^{(l)}\left(\xi_{3}, \xi_{3}\right)
\end{array}\right)
$$

we have the WFM formula,

$$
\begin{gathered}
\left(\begin{array}{cc}
-\mathbf{G}_{1-l, 1-l}^{(l)}\left(\xi_{1}, \xi_{1}\right) \overline{\mathbf{h}}^{L \dagger}-\psi_{1}^{\mathrm{ref},(l)}\left(\xi_{1}\right)\left(\psi_{0}^{\mathrm{ref}}\left(\xi_{3}\right)\right)^{-1} & -\mathbf{G}_{1-l, n+l}^{(l)}\left(\xi_{1}, \xi_{3}\right) \overline{\mathbf{h}}^{R} \\
-\mathbf{G}_{n+l, 1-l}^{(l)}\left(\xi_{3}, \xi_{1}\right) \overline{\mathbf{h}}^{L \dagger} & -\mathbf{G}_{n+l, n+l}^{(l)}\left(\xi_{3}, \xi_{3}\right) \overline{\mathbf{h}}^{R}-\psi_{n}^{(l)}\left(\xi_{3}\right)\left(\psi_{n+1}\left(\xi_{1}\right)\right)^{-1}
\end{array}\right) \\
\times\left(\begin{array}{c}
\psi_{0}^{\mathrm{ref}}\left(\xi_{3}\right) \\
\psi_{n+1}\left(\xi_{1}\right)
\end{array}\right)=\left(\begin{array}{c}
\mathbf{G}_{1-l, 1-l}^{(l)}\left(\xi_{1}, \xi_{1}\right) \overline{\mathbf{h}}^{L \dagger} \psi_{0}^{\mathrm{in}}\left(\xi_{3}\right)+\psi_{1}^{\mathrm{in},(l)}\left(\xi_{1}\right) \\
\mathbf{G}_{n+l, 1-l}^{(l)}\left(\xi_{3}, \xi_{1}\right) \overline{\mathbf{h}}^{L \dagger} \psi_{0}^{\mathrm{in}}\left(\xi_{3}\right)
\end{array}\right) .
\end{gathered}
$$

Applying the same separation using $\xi_{1}, \xi_{2}$, and $\xi_{3}$ to $\widetilde{\boldsymbol{\Phi}}_{R}^{+}$and $\widetilde{\boldsymbol{\Phi}}_{L}^{-}$, Eqs. (B9) and (B10) take the forms of

$$
\begin{gathered}
\psi_{n+1}\left(\xi_{3}\right)\left(\psi_{n+2}\left(\xi_{1}\right)\right)^{-1}=\widetilde{\boldsymbol{\Phi}}_{R}^{+}\left(\xi_{3}\right)\left(\widetilde{\boldsymbol{\Lambda}}_{R}^{+} \widetilde{\boldsymbol{\Phi}}_{R}^{+}\left(\xi_{1}\right)\right)^{-1}, \\
\psi_{0}^{\mathrm{ref}}\left(\xi_{1}\right)\left(\psi_{-1}^{\mathrm{ref}}\left(\xi_{3}\right)\right)^{-1}=\widetilde{\boldsymbol{\Phi}}_{L}^{-}\left(\xi_{1}\right)\left(\left(\widetilde{\boldsymbol{\Lambda}}_{L}^{-}\right)^{-1} \widetilde{\boldsymbol{\Phi}}_{L}^{-}\left(\xi_{3}\right)\right)^{-1},
\end{gathered}
$$

respectively. Since the rapidly decreasing evanescent waves are not included in $\widetilde{\boldsymbol{\Lambda}}_{R}^{+} \widetilde{\boldsymbol{\Phi}}_{R}^{+}\left(\xi_{1}\right)$ and $\left(\widetilde{\mathbf{\Lambda}}_{L}^{-}\right)^{-1} \widetilde{\mathbf{\Phi}}_{L}^{-}\left(\xi_{3}\right),\left(\widetilde{\boldsymbol{\Lambda}}_{R}^{+} \widetilde{\boldsymbol{\Phi}}_{R}^{+}\left(\xi_{1}\right)\right)$ and $\left(\left(\widetilde{\mathbf{\Lambda}}_{L}^{-}\right)^{-1} \widetilde{\boldsymbol{\Phi}}_{L}^{-}\left(\xi_{3}\right)\right)^{-1}$ are computed by the pseudoinverses. The ratio of the SWFs in Eq. (C5) is an $m_{L L} \times m_{L L}\left(m_{R R} \times m_{R R}\right)$ matrix, while that in Eq. (B7) is an $m_{L} \times m_{L}\left(m_{R} \times m_{R}\right)$ matrix. It is not possible to solve Eqs. (B12) and (B13) using $\psi_{0}^{\text {ref }}\left(\xi_{1}\right)\left(\psi_{-1}^{\text {ref }}\left(\xi_{3}\right)\right)^{-1}$ and $\psi_{n+1}\left(\xi_{3}\right)\left(\psi_{n+2}\left(\xi_{1}\right)\right)^{-1}$ strictly because $m_{L}>m_{L L}$ and $m_{R}>m_{R R}$. We derive other continued-fraction equations for the ratio of SWFs. $\psi_{1}^{\text {ref, }(l)}$ obeys the equation,

$$
\left(\begin{array}{c}
\psi_{0}^{\text {ref }}\left(\xi_{1}\right) \\
\psi_{0}^{\text {ref }}\left(\xi_{2}\right) \\
\psi_{0}^{\text {ref }}\left(\xi_{3}\right)
\end{array}\right)=\left(\begin{array}{lll}
\boldsymbol{\theta}_{L}\left(\xi_{1}, \xi_{1}\right) & \boldsymbol{\theta}_{L}\left(\xi_{1}, \xi_{2}\right) & \boldsymbol{\theta}_{L}\left(\xi_{1}, \xi_{3}\right) \\
\boldsymbol{\theta}_{L}\left(\xi_{2}, \xi_{1}\right) & \boldsymbol{\theta}_{L}\left(\xi_{2}, \xi_{2}\right) & \boldsymbol{\theta}_{L}\left(\xi_{2}, \xi_{3}\right) \\
\boldsymbol{\theta}_{L}\left(\xi_{3}, \xi_{1}\right) & \boldsymbol{\theta}_{L}\left(\xi_{3}, \xi_{2}\right) & \boldsymbol{\theta}_{L}\left(\xi_{3}, \xi_{3}\right)
\end{array}\right)\left(\begin{array}{c}
-\overline{\mathbf{h}}^{L \dagger} \psi_{-1}^{\mathrm{ref}}\left(\xi_{3}\right) \\
0 \\
-\overline{\mathbf{h}}^{L} \psi_{1}^{\mathrm{ref},(l)}\left(\xi_{1}\right)
\end{array}\right)
$$

where $\boldsymbol{\theta}_{L}\left(\xi_{i}, \xi_{j}\right)$ is the $(i, j)$ th block-matrix element of $\left(\overline{\mathbf{H}}_{L}\right)^{-1}$ defined in Eq. (A2). From the first and third block rows of Eq. (C8), we see that

$$
\begin{aligned}
& \psi_{0}^{\mathrm{ref}}\left(\xi_{1}\right)=-\boldsymbol{\theta}_{L}\left(\xi_{1}, \xi_{1}\right) \overline{\mathbf{h}}^{L \dagger} \psi_{-1}^{\mathrm{ref}}\left(\xi_{3}\right)-\boldsymbol{\theta}_{L}\left(\xi_{1}, \xi_{3}\right) \overline{\mathbf{h}}^{L} \psi_{1}^{\mathrm{ref},(l)}\left(\xi_{1}\right), \\
& \psi_{0}^{\mathrm{ref}}\left(\xi_{3}\right)=-\boldsymbol{\theta}_{L}\left(\xi_{3}, \xi_{1}\right) \overline{\mathbf{h}}^{L \dagger} \psi_{-1}^{\mathrm{ref}}\left(\xi_{3}\right)-\boldsymbol{\theta}_{L}\left(\xi_{3}, \xi_{3}\right) \overline{\mathbf{h}}^{L} \psi_{1}^{\mathrm{ref},(l)}\left(\xi_{1}\right) .
\end{aligned}
$$

Multiplying $\left(\psi_{-1}^{\text {ref }}\left(\xi_{3}\right)\right)^{-1}$ from the right side of Eq. (C9) leads to

$$
\psi_{0}^{\mathrm{ref}}\left(\xi_{1}\right)\left(\psi_{-1}^{\mathrm{ref}}\left(\xi_{3}\right)\right)^{-1}=-\boldsymbol{\theta}_{L}\left(\xi_{1}, \xi_{1}\right) \overline{\mathbf{h}}^{L \dagger}-\boldsymbol{\theta}_{L}\left(\xi_{1}, \xi_{3}\right) \overline{\mathbf{h}}^{L} \psi_{1}^{\mathrm{ref},(l)}\left(\xi_{1}\right)\left(\psi_{-1}^{\mathrm{ref}}\left(\xi_{3}\right)\right)^{-1} .
$$

Multiplying $\left(\psi_{1}^{\text {ref, }(l)}\left(\xi_{1}\right)\right)^{-1}$ from the right side of Eq. (C10), we have

$$
\psi_{0}^{\mathrm{ref}}\left(\xi_{3}\right)\left(\psi_{1}^{\mathrm{ref},(l)}\left(\xi_{1}\right)\right)^{-1}=-\boldsymbol{\theta}_{L}\left(\xi_{3}, \xi_{1}\right) \overline{\mathbf{h}}^{L \dagger} \psi_{-1}^{\mathrm{ref}}\left(\xi_{3}\right)\left(\psi_{1}^{\mathrm{ref},(l)}\left(\xi_{1}\right)\right)^{-1}-\boldsymbol{\theta}_{L}\left(\xi_{3}, \xi_{3}\right) \overline{\mathbf{h}}^{L} .
$$

Substituting $\psi_{1}^{\text {ref, }(l)}\left(\xi_{1}\right)\left(\psi_{-1}^{\text {ref }}\left(\xi_{3}\right)\right)^{-1}$ in Eq. $(\mathrm{C} 11)$ into $\psi_{-1}^{\text {ref }}\left(\xi_{3}\right)\left(\psi_{1}^{\text {ref, }(l)}\left(\xi_{1}\right)\right)^{-1}$ in Eq. (C12), we obtain the continued-fraction equation for the left electrode side,

$$
\psi_{0}^{\text {ref }}\left(\xi_{3}\right)\left(\psi_{1}^{\text {ref },(l)}\left(\xi_{1}\right)\right)^{-1}=-\boldsymbol{\theta}_{L}\left(\xi_{3}, \xi_{3}\right) \overline{\mathbf{h}}^{L}+\boldsymbol{\theta}_{L}\left(\xi_{3}, \xi_{1}\right) \overline{\mathbf{h}}^{L \dagger}\left(\psi_{0}^{\text {ref }}\left(\xi_{1}\right)\left(\psi_{-1}^{\text {ref }}\left(\xi_{3}\right)\right)^{-1}+\boldsymbol{\theta}_{L}\left(\xi_{1}, \xi_{1}\right) \overline{\mathbf{h}}^{L \dagger}\right)^{-1} \boldsymbol{\theta}_{L}\left(\xi_{1}, \xi_{3}\right) \overline{\mathbf{h}}^{L}
$$


Analogous to Eq. (C13), the continued-fraction equation for the right electrode side is given by

$$
\psi_{n+1}\left(\xi_{1}\right)\left(\psi_{n}^{(l)}\left(\xi_{3}\right)\right)^{-1}=-\boldsymbol{\theta}_{R}\left(\xi_{1}, \xi_{1}\right) \overline{\mathbf{h}}^{R \dagger}+\boldsymbol{\theta}_{R}\left(\xi_{1}, \xi_{3}\right) \overline{\mathbf{h}}^{R}\left(\psi_{n+1}\left(\xi_{3}\right)\left(\psi_{n+2}\left(\xi_{1}\right)\right)^{-1}+\boldsymbol{\theta}_{R}\left(\xi_{3}, \xi_{3}\right) \overline{\mathbf{h}}^{R}\right)^{-1} \boldsymbol{\theta}_{R}\left(\xi_{3}, \xi_{1}\right) \overline{\mathbf{h}}^{R \dagger}
$$

Shifting the matching planes by one extra layer inside of the transition region as discussed in Sec. IIE, we obtain the following WFM formula in which the left (right) matching plane is set between $\psi_{1}^{(l)}\left(\xi_{3}\right)$ and $\psi_{1}^{(l-1)}\left(\xi_{1}\right)\left[\psi_{n}^{(l)}\left(\xi_{1}\right)\right.$ and $\left.\psi_{n}^{(l-1)}\left(\xi_{3}\right)\right]$.

$$
\begin{gathered}
\left(\begin{array}{cc}
-\mathbf{G}_{2-l, 2-l}^{(l-1)}\left(\xi_{1}, \xi_{1}\right) \overline{\mathbf{h}}^{L \dagger}-\psi_{1}^{\mathrm{ref},(l-1)}\left(\xi_{1}\right)\left(\psi_{1}^{\mathrm{ref},(l)}\left(\xi_{3}\right)\right)^{-1} & -\mathbf{G}_{2-l, n+l-1}^{(l-1)}\left(\xi_{1}, \xi_{3}\right) \overline{\mathbf{h}}^{R} \\
-\mathbf{G}_{n+l-1,2-l}^{(l-1)}\left(\xi_{3}, \xi_{1}\right) \overline{\mathbf{h}}^{L \dagger} & -\mathbf{G}_{n+l-1, n+l-1}^{(l-1)}\left(\xi_{3}, \xi_{3}\right) \overline{\mathbf{h}}^{R}-\psi_{n}^{(l-1)}\left(\xi_{3}\right)\left(\psi_{n}^{(l)}\left(\xi_{1}\right)\right)^{-1}
\end{array}\right)\left(\begin{array}{c}
\psi_{1}^{\mathrm{ref},(l)}\left(\xi_{3}\right) \\
\psi_{n}^{(l)}\left(\xi_{1}\right)
\end{array}\right) \\
=\left(\begin{array}{c}
\mathbf{G}_{2-l, 2-l}^{(l-1)}\left(\xi_{1}, \xi_{1}\right) \overline{\mathbf{h}}^{L \dagger} \psi_{1}^{\mathrm{in},(l)}\left(\xi_{3}\right)+\psi_{1}^{\mathrm{in},(l-1)}\left(\xi_{1}\right) \\
\mathbf{G}_{n+l-1,2-l}^{(l-1)}\left(\xi_{3}, \xi_{1}\right) \overline{\mathbf{h}}^{L \dagger} \psi_{1}^{\text {in, },(l)}\left(\xi_{3}\right)
\end{array}\right) .
\end{gathered}
$$

By repeatedly solving Eqs. (C13) and (C14) $l$ times, the WFM formula is rewritten as

$$
\begin{gathered}
\left(\begin{array}{cc}
-\mathbf{G}_{1,1}^{(0)}\left(\xi_{1}, \xi_{1}\right) \overline{\mathbf{h}}^{L \dagger}-\psi_{1}^{\mathrm{ref},(0)}\left(\xi_{1}\right)\left(\psi_{1}^{\mathrm{ref},(1)}\left(\xi_{3}\right)\right)^{-1} & -\mathbf{G}_{1, n}^{(0)}\left(\xi_{1}, \xi_{3}\right) \overline{\mathbf{h}}^{R} \\
-\mathbf{G}_{n, 1}^{(0)}\left(\xi_{3}, \xi_{1}\right) \overline{\mathbf{h}}^{L \dagger} & -\mathbf{G}_{n, n}^{(0)}\left(\xi_{3}, \xi_{3}\right) \overline{\mathbf{h}}^{R}-\psi_{n}^{(0)}\left(\xi_{3}\right)\left(\psi_{n}^{(1)}\left(\xi_{1}\right)\right)^{-1}
\end{array}\right)\left(\begin{array}{c}
\psi_{1}^{\mathrm{ref},(1)}\left(\xi_{3}\right) \\
\psi_{n}^{(1)}\left(\xi_{1}\right)
\end{array}\right) \\
=\left(\begin{array}{c}
\mathbf{G}_{1,1}^{(0)}\left(\xi_{1}, \xi_{1}\right) \overline{\mathbf{h}}^{L \dagger} \psi_{1}^{\mathrm{in},(1)}\left(\xi_{3}\right)+\psi_{1}^{\mathrm{in},(0)}\left(\xi_{1}\right) \\
\mathbf{G}_{n, 1}^{(0)}\left(\xi_{3}, \xi_{1}\right) \overline{\mathbf{h}}^{L \dagger} \psi_{1}^{\mathrm{in},(1)}\left(\xi_{3}\right)
\end{array}\right)
\end{gathered}
$$

Defining the $m_{L L} \times m_{L L}$ and $m_{R R} \times m_{R R}$ ratio matrices for the left and right electrode sides,

$$
\begin{gathered}
\mathbf{R}^{\mathrm{ref},(l-1)}=\psi_{1}^{\mathrm{ref},(l)}\left(\xi_{3}\right)\left(\psi_{1}^{\mathrm{ref},(l-1)}\left(\xi_{1}\right)\right)^{-1}, \\
\mathbf{R}^{\operatorname{tra},(l)}=\psi_{n}^{(l)}\left(\xi_{1}\right)\left(\psi_{n}^{(l-1)}\left(\xi_{3}\right)\right)^{-1},
\end{gathered}
$$

respectively, Eqs. (C13), (C14), and (C16) are expressed as

$$
\begin{aligned}
& \mathbf{R}^{\mathrm{ref},(l-1)}=-\boldsymbol{\theta}_{L}\left(\xi_{3}, \xi_{3}\right) \overline{\mathbf{h}}^{L}+\boldsymbol{\theta}_{L}\left(\xi_{3}, \xi_{1}\right) \overline{\mathbf{h}}^{L \dagger}\left(\left(\mathbf{R}^{\mathrm{ref},(l)}\right)^{-1}+\boldsymbol{\theta}_{L}\left(\xi_{1}, \xi_{1}\right) \overline{\mathbf{h}}^{L \dagger}\right)^{-1} \boldsymbol{\theta}_{L}\left(\xi_{1}, \xi_{3}\right) \overline{\mathbf{h}}^{L}, \\
& \mathbf{R}^{\operatorname{tra},(l)}=-\boldsymbol{\theta}_{R}\left(\xi_{1}, \xi_{1}\right) \overline{\mathbf{h}}^{R \dagger}+\boldsymbol{\theta}_{R}\left(\xi_{1}, \xi_{3}\right) \overline{\mathbf{h}}^{R}\left(\left(\mathbf{R}^{\operatorname{tra},(l+1)}\right)^{-1}+\boldsymbol{\theta}_{R}\left(\xi_{3}, \xi_{3}\right) \overline{\mathbf{h}}^{R}\right)^{-1} \boldsymbol{\theta}_{R}\left(\xi_{3}, \xi_{1}\right) \overline{\mathbf{h}}^{R \dagger}, \\
& \left(\begin{array}{cc}
-\mathbf{G}_{1,1}^{(0)}\left(\xi_{1}, \xi_{1}\right) \overline{\mathbf{h}}^{L \dagger}-\left(\mathbf{R}^{\mathrm{ref},(0)}\right)^{-1} & -\mathbf{G}_{1, n}^{(0)}\left(\xi_{1}, \xi_{3}\right) \overline{\mathbf{h}}^{R} \\
-\mathbf{G}_{n, 1}^{(0)}\left(\xi_{3}, \xi_{1}\right) \overline{\mathbf{h}}^{L \dagger} & -\mathbf{G}_{n, n}^{(0)}\left(\xi_{3}, \xi_{3}\right) \overline{\mathbf{h}}^{R}-\left(\mathbf{R}^{\mathrm{tra},(1)}\right)^{-1}
\end{array}\right)\left(\begin{array}{c}
\psi_{1}^{\mathrm{ref},(1)}\left(\xi_{3}\right) \\
\psi_{n}^{(1)}\left(\xi_{1}\right)
\end{array}\right)=\left(\begin{array}{c}
\mathbf{G}_{1,1}^{(0)}\left(\xi_{1}, \xi_{1}\right) \overline{\mathbf{h}}^{L \dagger} \psi_{1}^{\mathrm{in},(1)}\left(\xi_{3}\right)+\psi_{1}^{\mathrm{in},(0)}\left(\xi_{1}\right) \\
\mathbf{G}_{n, 1}^{(0)}\left(\xi_{3}, \xi_{1}\right) \overline{\mathbf{h}}^{L \dagger} \psi_{1}^{\mathrm{in},(1)}\left(\xi_{3}\right)
\end{array}\right) .
\end{aligned}
$$

From the discussion in Sec. IIE, the ratio matrices are uniquely determined if $l$ is sufficiently large. Inserting $\psi_{1}^{\text {ref,(1) }}\left(\xi_{3}\right)$ and $\psi_{n}^{(1)}\left(\xi_{1}\right)$ into Eq. (C3), the SWFs in the transition region are computed.

For the computation of the transmission probability, the matrices containing the group velocities of the incident and transmitted waves and the vector of the transmission coefficients are given by

$$
\begin{aligned}
\mathbf{v}^{\text {in }} & =a \boldsymbol{\Phi}_{L}^{+\dagger}\left(\xi_{1}\right) \boldsymbol{\Gamma}_{L}^{(0)} \boldsymbol{\Phi}_{L}^{+}\left(\xi_{1}\right), \\
\check{\mathbf{v}}^{\text {tra }} & =a\left(\check{\boldsymbol{\Phi}}_{R}^{+}\right)^{\dagger}\left(\xi_{1}\right) \boldsymbol{\Gamma}_{R}^{(0)} \check{\boldsymbol{\Phi}}_{R}^{+}\left(\xi_{1}\right), \\
\check{\mathbf{t}}_{k} & =\left(\check{\boldsymbol{\Phi}}_{R}^{+}\left(\xi_{1}\right)\right)^{-1} \psi_{n, k}^{(1)}\left(\xi_{1}\right),
\end{aligned}
$$

respectively. The transmission matrix is obtained by

$$
T_{k, k}=a\left(\mathbf{v}_{k, k}^{\text {in }}\right)^{-1} \psi_{n, k}^{(1) \dagger}\left(\xi_{1}\right) \Gamma_{R}^{(0)} \psi_{n, k}^{(1)}\left(\xi_{1}\right)
$$

with the dimension of $\boldsymbol{\Gamma}_{R}^{(0)}$ being $m_{R R}$. 


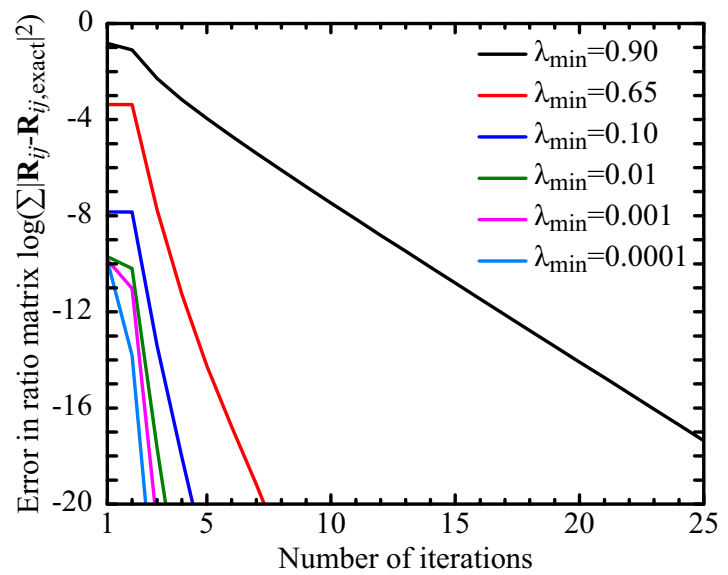

FIG. 11. Convergence behavior of the ratio matrix $\mathbf{R}^{\text {ref }}$.

\section{APPENDIX D: CONVERGENCE BEHAVIOR OF THE RATIO MATRIX}

As introduced in Sec. IIE, the ratio matrices $\mathbf{R}^{\text {ref,(0) }}$ and $\mathbf{R}^{\text {tra,(1) }}$ are obtained by solving the continued-fraction equations Eqs. (27) and (28). The initial matrix of $\left(\widetilde{\boldsymbol{\Lambda}}_{L}^{-}\right)^{-1} \widetilde{\boldsymbol{\Phi}}_{L}^{-}\left(\widetilde{\boldsymbol{\Phi}}_{L}^{-}\right)^{-1}\left[\left(\widetilde{\boldsymbol{\Lambda}}_{R}^{+}\right)^{-1} \widetilde{\boldsymbol{\Phi}}_{R}^{+}\left(\widetilde{\boldsymbol{\Phi}}_{R}^{+}\right)^{-1}\right]$ is constructed at $\psi_{-1}$ and $\psi_{0}\left(\psi_{n+1}\right.$ and $\left.\psi_{n+2}\right)$, in which the rapidly decreasing evanescent waves vanish. It is of importance that the ratio matrix $\mathbf{R}^{\text {ref,(0) }}\left(\mathbf{R}^{\text {tra,(1) }}\right)$ is uniquely determined after the continuedfraction equation Eq. (27) [Eq. (28)] is solved until $\mathbf{R}^{\text {ref,(0) }}$ $\left(\mathbf{R}^{\text {tra,(1) }}\right)$ on the left- and right-hand sides become identical. Figure 11 shows the convergence behavior of the ratio matrix $\mathbf{R}^{\text {ref }}$ for the $(9,0)$ CNT electrode used in Sec. III A as the electrode region. $\mathbf{R}_{\text {exact }}^{\text {ref }}$ are computed by solving Eq. (C19) 10 times using the initial value of $\left(\widetilde{\boldsymbol{\Lambda}}_{L}^{-}\right)^{-1} \widetilde{\boldsymbol{\Phi}}_{L}^{-}\left(\widetilde{\boldsymbol{\Phi}}_{L}^{-}\right)^{-1}$ with $\lambda_{\text {min }}=10^{-10}$ as a reference and the difference from $\mathbf{R}_{\text {exact }}^{\text {ref }}$ are plotted in Fig. 11. We can see that the number of iterations for solving the continued-fraction equations is small when $\lambda_{\text {min }}$ is set to be small and the ratio matrices can be uniquely determined by the continued-fraction equations.

\section{APPENDIX E: DETERIORATION OF THE TRANSLATIONAL INVARIANCE OF THE TRANSMISSION PROBABILITY DUE TO THE PSEUDOINVERSE}

The transmission coefficient should not change except the trivial Bloch factors as long as the same matrices for the group velocity are used to retain the translational invariance of the transmission probability. The transmission coefficients at the first and second extra layers from the boundary between the right electrode and transition regions, $\tilde{\boldsymbol{t}}_{n, k}^{(l)}$ and $\tilde{\boldsymbol{t}}_{n, k}^{(l-1)}$, should satisfy the following relation,

$$
\widetilde{\boldsymbol{t}}_{n, k}^{(l)}=\widetilde{\boldsymbol{\Lambda}}_{R}^{+} \widetilde{\boldsymbol{t}}_{n, k}^{(l-1)} .
$$

According to Eqs. (16) and (18), the SWFs at the first and second extra layers are described as

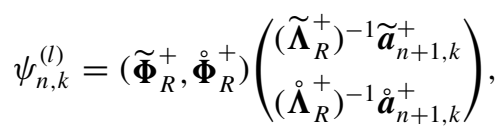

$$
\psi_{n, k}^{(l-1)}=\left(\widetilde{\boldsymbol{\Phi}}_{R}^{+}, \stackrel{\boldsymbol{\Phi}}{R}_{R}^{+}\right)\left(\begin{array}{c}
\left(\widetilde{\boldsymbol{\Lambda}}_{R}^{+}\right)^{-2} \widetilde{\boldsymbol{a}}_{n+1, k}^{+} \\
\left(\stackrel{\boldsymbol{\Lambda}}{R}_{R}^{+}\right)^{-2} \stackrel{\circ}{\boldsymbol{a}}_{n+1, k}^{+}
\end{array}\right),
$$

respectively. Note that in the transition region, the evanescent waves excluded by the cutoff parameter $\lambda_{\min }$ also contribute to the SWFs. Multiplying the pseudoinverse $\left(\widetilde{\boldsymbol{\Phi}}_{R}^{+}\right)^{-1}$ to Eqs. (E2) and (E3) from the left hand side leads to

$$
\begin{aligned}
& \widetilde{\boldsymbol{t}}_{n, k}^{(l)}=\left(\widetilde{\boldsymbol{\Phi}}_{R}^{+}\right)^{-1}\left(\widetilde{\boldsymbol{\Phi}}_{R}^{+}, \stackrel{\stackrel{\boldsymbol{\Phi}}{ }}{R}^{+}\right)\left(\begin{array}{c}
\left(\widetilde{\boldsymbol{\Lambda}}_{R}^{+}\right)^{-1} \widetilde{\boldsymbol{a}}_{n+1, k}^{+} \\
\left(\stackrel{\boldsymbol{\Lambda}}{R}^{+}\right)^{-1} \stackrel{\stackrel{\boldsymbol{a}}{ }}{n+1, k}^{+}
\end{array}\right) \\
& =\left(\widetilde{\boldsymbol{\Lambda}}_{R}^{+}\right)^{-1} \widetilde{\boldsymbol{a}}_{n+1, k}^{+}+\left(\widetilde{\boldsymbol{\Phi}}_{R}^{+}\right)^{-1} \stackrel{\circ}{\boldsymbol{\Phi}}_{R}^{+}\left(\stackrel{\circ}{\boldsymbol{\Lambda}}_{R}^{+}\right)^{-1} \stackrel{\mathrm{a}}{n+1, k}_{n+}^{+},
\end{aligned}
$$

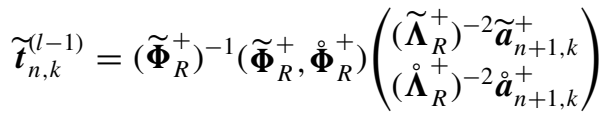

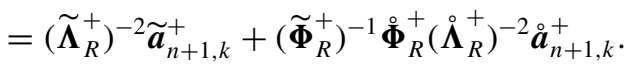

Since the generalized Bloch waves are nonorthogonal, $\left(\widetilde{\boldsymbol{\Phi}}_{R}^{+}\right)^{-1} \stackrel{\circ}{\boldsymbol{\Phi}}_{R}^{+} \neq \mathbf{0}$, resulting in $\widetilde{\mathbf{t}}_{n, k}^{(l)} \neq \widetilde{\boldsymbol{\Lambda}}_{R}^{+} \widetilde{\mathbf{t}}_{n, k}^{(l-1)}$. When a regular matrix is used for $\check{\boldsymbol{\Phi}}_{R}^{+}$, the second terms of Eqs. (E4) and (E5) vanish and the translational invariance is preserved.

\section{APPENDIX F: INVARIANCE OF THE TRANSMISSION MATRIX WITH RESPECT TO THE TRANSMITTED WAVES}

We prove Eq. (53) indicating that the transmission matrix is invariant as long as a regular matrix is taken for $\check{\boldsymbol{\Phi}}_{R}^{+}$. Let the number of columns of $\widetilde{\boldsymbol{\Phi}}_{R}^{+}\left(\stackrel{\circ}{\boldsymbol{\Phi}}_{R}^{+}\right)$be $\widetilde{m}_{R}\left(\stackrel{\circ}{m}_{R}\right)$. Defining a $m_{R} \times m_{R}$ regular matrix

$$
\mathbf{P}=\left(\begin{array}{ll}
\mathbf{I} & \mathbf{p}_{1} \\
\mathbf{0} & \mathbf{p}_{2}
\end{array}\right)
$$

with $\mathbf{p}_{1}$ and $\mathbf{p}_{2}$ being $\tilde{m}_{R} \times \stackrel{\circ}{m}_{R}$ and $\stackrel{\circ}{m}_{R} \times \stackrel{\circ}{m}_{R}$ matrices, respectively, $\boldsymbol{\Phi}_{R}^{+}$and $\check{\boldsymbol{\Phi}}_{R}^{+}$are related as

$$
\boldsymbol{\Phi}_{R}^{+}=\check{\boldsymbol{\Phi}}_{R}^{+} \mathbf{P} \text {. }
$$

From Eq. (F1), one sees that $\check{\boldsymbol{\Phi}}_{R}^{+}$can include the components of $\widetilde{\boldsymbol{\Phi}}_{R}^{+}$. Equation (51) is rewritten as

$$
\begin{aligned}
\check{\boldsymbol{t}}_{k} & =\left(\check{\boldsymbol{\Phi}}_{R}^{+}\right)^{-1} \psi_{n, k}^{(1)} \\
& =\mathbf{P}\left(\boldsymbol{\Phi}_{R}^{+}\right)^{-1} \psi_{n, k}^{(1)} \\
& =\mathbf{P} \mathbf{t}_{k} .
\end{aligned}
$$

Then, we have

$$
\check{\mathbf{t}}=\mathbf{P t} \text {. }
$$

The group velocity of the transmitted wave is expressed as

$$
\begin{aligned}
\check{\mathbf{v}}^{\text {tra }} & =a\left(\check{\boldsymbol{\Phi}}_{R}^{+}\right)^{\dagger} \boldsymbol{\Gamma}_{R}^{(0)} \check{\boldsymbol{\Phi}}_{R}^{+} \\
& =a\left(\boldsymbol{\Phi}_{R}^{+} \mathbf{P}^{-1}\right)^{\dagger} \boldsymbol{\Gamma}_{R}^{(0)} \boldsymbol{\Phi}_{R}^{+} \mathbf{P}^{-1} \\
& =\left(\mathbf{P}^{-1}\right)^{\dagger} \mathbf{v}^{\operatorname{tra}} \mathbf{P}^{-1}
\end{aligned}
$$


Substituting Eqs. (F2) and (F3) into Eq. (40) leads to

$$
\begin{aligned}
\mathbf{T} & =\left(\mathbf{v}^{\text {in }}\right)^{-1} \check{\mathbf{t}}^{\dagger} \check{\mathbf{v}}^{\operatorname{tra} \check{\mathbf{t}}} \\
& =\left(\mathbf{v}^{\text {in }}\right)^{-1}(\mathbf{P t})^{\dagger}\left(\mathbf{P}^{-1}\right)^{\dagger} \mathbf{v}^{\text {tra }} \mathbf{P}^{-1} \mathbf{P t} \\
& =\left(\mathbf{v}^{\text {in }}\right)^{-1} \mathbf{t}^{\dagger} \mathbf{v}^{\operatorname{tra}} \mathbf{t} .
\end{aligned}
$$

\section{APPENDIX G: WAVE-FUNCTION-MATCHING FORMULA WITHOUT GREEN'S FUNCTION}

Computation of the Green's function of the isolated transition region $\widehat{\mathbf{G}}^{(l)}$ is time consuming in some cases. In this Appendix, we introduce an alternative procedure which solves simultaneous equations for the SWFs [55]. The SWFs in the electrode regions are rewritten as

$$
\begin{gathered}
\psi_{0}=\psi_{0}^{\mathrm{ref}}+\psi_{0}^{\mathrm{in}} \\
=\psi_{0}^{\mathrm{ref}}\left(\psi_{1}^{\mathrm{ref},(l)}\right)^{-1} \psi_{1}^{\mathrm{ref},(l)}+\psi_{0}^{\mathrm{in}} \\
=\mathbf{R}^{\mathrm{ref},(l)}\left(\psi_{1}^{(l)}-\psi_{1}^{\mathrm{in},(l)}\right)+\psi_{0}^{\mathrm{in}}, \\
\psi_{n+1}=\psi_{n+1}\left(\psi_{n}^{(l)}\right)^{-1} \psi_{n}^{(l)} \\
=\mathbf{R}^{\mathrm{rra},(l)} \psi_{n}^{(l)} .
\end{gathered}
$$

Inserting Eqs. (45), (46), (G1), and (G2) into Eq. (B2) yields [55]

$$
\begin{gathered}
\left(E \widehat{\mathbf{S}}^{(l)}-\widehat{\mathbf{H}}^{(l)}-\widetilde{\mathbf{H}}^{(l)}\right)\left(\begin{array}{c}
\psi_{1}^{(l)} \\
\vdots \\
\vdots \\
\vdots \\
\psi_{n}^{(l)}
\end{array}\right) \\
=\left(\begin{array}{c}
-\overline{\mathbf{H}}_{L, L}^{\dagger} \psi_{0}^{\text {in }}-\boldsymbol{\Sigma}_{L}^{(l)} \psi_{1}^{\text {in, },(l)} \\
0 \\
\vdots \\
0 \\
0
\end{array}\right),
\end{gathered}
$$

where $\widetilde{\mathbf{H}}^{(l)}$ is a zero matrix except that the upper left $m_{L L} \times$ $m_{L L}$ and lower right $m_{R R} \times m_{R R}$ block-matrix elements are $\Sigma_{L}^{(l)}$ and $\Sigma_{R}^{(l)}$, respectively:

$$
\widetilde{\mathbf{H}}^{(l)}=\left(\begin{array}{ccccc}
\boldsymbol{\Sigma}_{L}^{(l)} & 0 & \ldots & \ldots & 0 \\
0 & 0 & & & \vdots \\
\vdots & & \ddots & & \vdots \\
\vdots & & & 0 & 0 \\
0 & \ldots & \ldots & 0 & \Sigma_{R}^{(l)}
\end{array}\right) .
$$

The SWFs can be evaluated without computing the Green's functions of the isolated transition region $\widehat{\mathbf{G}}^{(l)}$ if one solves Eq. (G3). Inserting $\psi_{1}^{(l)}$ and $\psi_{n}^{(l)}$ into Eqs. (G1) and (G2), one has the SWFs at the first layers of the electrode regions $\psi_{0}^{\text {ref }}\left(=\psi_{0}-\psi_{0}^{\text {in }}\right)$ and $\psi_{n+1}$, which are used to compute the transmission and reflection coefficients.
When the matching planes are moved, Eq. (G3) is rewritten as

$$
\begin{gathered}
\left(E \widehat{\mathbf{S}}^{(0)}-\widehat{\mathbf{H}}^{(0)}-\widetilde{\mathbf{H}}^{(0)}\right)\left(\begin{array}{c}
\psi_{1}^{(0)} \\
\vdots \\
\vdots \\
\vdots \\
\psi_{n}^{(0)}
\end{array}\right) \\
=\left(\begin{array}{c}
-\overline{\mathbf{H}}_{L, L}^{\dagger} \psi_{1}^{\text {in, }(1)}-\Sigma_{L}^{(0)} \psi_{1}^{\text {in, },(0)} \\
0 \\
\vdots \\
0 \\
0
\end{array}\right),
\end{gathered}
$$

where

$$
\widetilde{\mathbf{H}}^{(0)}=\left(\begin{array}{ccccc}
\Sigma_{L}^{(0)} & 0 & \ldots & \ldots & 0 \\
0 & 0 & & & \vdots \\
\vdots & & \ddots & & \vdots \\
\vdots & & & 0 & 0 \\
0 & \ldots & \ldots & 0 & \Sigma_{R}^{(0)}
\end{array}\right) .
$$

In the case that $m_{L}>m_{L L}$ and $m_{R}>m_{R R}$, the self-energy terms are defined by

$$
\begin{gathered}
\boldsymbol{\Sigma}_{L}^{(l)}=-\overline{\mathbf{h}}^{L \dagger} \mathbf{R}^{\mathrm{ref},(l)}, \\
\boldsymbol{\Sigma}_{R}^{(l)}=-\overline{\mathbf{h}}^{R} \mathbf{R}^{\mathrm{tra},(l+1)} .
\end{gathered}
$$

Then, the similar equation with Eq. (G5) becomes

$$
\begin{gathered}
\left(E \widehat{\mathbf{S}}^{(0)}-\widehat{\mathbf{H}}^{(0)}-\widetilde{\mathbf{H}}^{(0)}\right)\left(\begin{array}{c}
\psi_{1}^{(0)}\left(\xi_{1}\right) \\
\vdots \\
\vdots \\
\vdots \\
\psi_{n}^{(0)}\left(\xi_{3}\right)
\end{array}\right) \\
=\left(\begin{array}{c}
-\overline{\mathbf{h}}^{L \dagger} \psi_{1}^{\text {in, },(1)}\left(\xi_{3}\right)-\Sigma_{L}^{(0)} \psi_{1}^{\text {in, },(0)}\left(\xi_{1}\right) \\
0 \\
\vdots \\
0 \\
0
\end{array}\right)
\end{gathered}
$$

where

$$
\widetilde{\mathbf{H}}^{(0)}=\left(\begin{array}{ccccc}
\Sigma_{L}^{(0)} & 0 & \ldots & \ldots & 0 \\
0 & 0 & & & \vdots \\
\vdots & & \ddots & & \vdots \\
\vdots & & & 0 & 0 \\
0 & \ldots & \ldots & 0 & \Sigma_{R}^{(0)}
\end{array}\right) .
$$

Here $\Sigma_{L}^{(0)}$ and $\boldsymbol{\Sigma}_{R}^{(0)}$ are $m_{L L} \times m_{L L}$ and $m_{R R} \times m_{R R}$ block matrices. 
[1] M. Brandbyge, J.-L. Mozos, P. Ordejón, J. Taylor, and K. Stokbro, Phys. Rev. B 65, 165401 (2002).

[2] S. Sanvito, C. J. Lambert, J. H. Jefferson, and A. M. Bratkovsky, Phys. Rev. B 59, 11936 (1999).

[3] J. Taylor, H. Guo, and J. Wang, Phys. Rev. B 63, 245407 (2001).

[4] H. H. B. Sørensen, P. C. Hansen, D. E. Petersen, S. Skelboe, and K. Stokbro, Phys. Rev. B 77, 155301 (2008).

[5] L. V. Keldysh, Zh. Eksp. Teor. Fiz. 47, 1515 (1965) [Sov. Phys. JETP 20, 1018 (1965)].

[6] T. Ando, Phys. Rev. B 44, 8017 (1991).

[7] N. D. Lang, Phys. Rev. B 52, 5335 (1995).

[8] K. Hirose and M. Tsukada, Phys. Rev. B 51, 5278 (1995).

[9] H. J. Choi and J. Ihm, Phys. Rev. B 59, 2267 (1999).

[10] S. Tsukamoto and K. Hirose, Phys. Rev. B 66, 161402 (2002).

[11] Y. Fujimoto and K. Hirose, Phys. Rev. B 67, 195315 (2003).

[12] K. Hirose, T. Ono, Y. Fujimoto, and S. Tsukamoto, First Principles Calculations in Real-Space Formalism, Electronic Configurations and Transport Properties of Nanostructures (Imperial College, London, 2005).

[13] P. A. Khomyakov and G. Brocks, Phys. Rev. B 70, 195402 (2004).

[14] T. Ono, Y. Egami, and K. Hirose, Phys. Rev. B 86, 195406 (2012).

[15] T. Ono and S. Tsukamoto, Phys. Rev. B 93, 045421 (2016).

[16] D. S. Fisher and P. A. Lee, Phys. Rev. B 23, 6851 (1981).

[17] M. Büttiker, Y. Imry, R. Landauer, and S. Pinhas, Phys. Rev. B 31, 6207 (1985).

[18] H. H. B. Sørensen, P. C. Hansen, D. E. Petersen, S. Skelboe, and K. Stokbro, Phys. Rev. B 79, 205322 (2009).

[19] S. Brück, M. Calderara, M. H. Bani-Hashemian, J. VandeVondele, and M. Luisier, J. Chem. Phys. 147, 074116 (2017).

[20] S. Iwase, Y. Futamura, A. Imakura, T. Sakurai, S. Tsukamoto, and T. Ono, arXiv:1709.09324.

[21] P. S. Krstić, X.-G. Zhang, and W. H. Butler, Phys. Rev. B 66, 205319 (2002).

[22] See Supplemental Material at http://link.aps.org/supplemental/ 10.1103/PhysRevB.97.115450 for the algorithm of the present method, the error evaluation at the energy where the number of propagating modes changes, and the error in the sum of the transmission and reflection probabilities of Model 1a.

[23] N. Troullier and J. L. Martins, Phys. Rev. B 43, 1993 (1991).

[24] S. Tsukamoto, K. Hirose, and S. Blügel, Phys. Rev. E 90, 013306 (2014).

[25] S. Tsukamoto, T. Ono, K. Hirose, and S. Blügel, Phys. Rev. E 95, 033309 (2017).

[26] S. E. Laux, Phys. Rev. B 86, 075103 (2012).

[27] S. Iwase, Y. Futamura, A. Imakura, T. Sakurai, and T. Ono, in Proceedings of 2017 International Conference for High Performance Computing, Networking, Storage and Analysis, SC ' 17 (ACM, New York, 2017).
[28] T. Sakurai and H. Sugiura, J. Comput. Appl. Math. 159, 119 (2003).

[29] We used the norm-conserving pseudopotentials NCPS97 constructed by K. Kobayashi. See K. Kobayashi, Comput. Mater. Sci. 14, 72 (1999).

[30] J. P. Perdew and A. Zunger, Phys. Rev. B 23, 5048 (1981).

[31] W. Kohn, Rev. Mod. Phys. 71, 1253 (1999).

[32] P. Hohenberg and W. Kohn, Phys. Rev. 136, B864 (1964).

[33] T. Ono and K. Hirose, Phys. Rev. Lett. 82, 5016 (1999).

[34] T. Ono and K. Hirose, Phys. Rev. B 72, 085115 (2005).

[35] T. Ono, M. Heide, N. Atodiresei, P. Baumeister, S. Tsukamoto, and S. Blügel, Phys. Rev. B 82, 205115 (2010).

[36] J. R. Chelikowsky, N. Troullier, and Y. Saad, Phys. Rev. Lett. 72, 1240 (1994).

[37] J. R. Chelikowsky, N. Troullier, K. Wu, and Y. Saad, Phys. Rev. B 50, 11355 (1994).

[38] M. I. Katsnelson, Graphene: Carbon in Two Dimensions (Cambridge University Press, Cambridge, 2012).

[39] S. Das Sarma, S. Adam, E. H. Hwang, and E. Rossi, Rev. Mod. Phys. 83, 407 (2011).

[40] A. H. Castro Neto, F. Guinea, N. M. R. Peres, K. S. Novoselov, and A. K. Geim, Rev. Mod. Phys. 81, 109 (2009).

[41] A. K. Geim and K. S. Novoselov, Nat. Mater. 6, 183 (2007).

[42] O. V. Yazyev and S. G. Louie, Nat. Mater. 9, 806 (2010).

[43] Y. Gong, G. Shi, Z. Zhang, W. Zhou, J. Jung, W. Gao, L. Ma, Y. Yang, S. Yang, G. You, R. Vajtai, Q. Xu, A. H. MacDonald, B. I. Yakobson, J. Lou, Z. Liu, and P. M. Ajayan, Nat. Commun. 5, 3193 (2014).

[44] K. Kim, H.-B.-R. Lee, R. W. Johnson, J. T. Tanskanen, N. Liu, M.-G. Kim, C. Pang, C. Ahn, S. F. Bent, and Z. Bao, Nat. Commun. 5, 4781 (2014).

[45] D. Berger and C. Ratsch, Phys. Rev. B 93, 235441 (2016).

[46] A. Suzuki, M. Tanabe, and S. Fujita, J. Mod. Phys. 8, 607 (2017).

[47] Throughout this section, the wave number $k_{x(z)}$ is normalized by $2 \pi / L_{x(z)}$, where $L_{x(z)}$ denotes the length of the transition region in the $x(z)$ direction.

[48] W. Kohn and L. J. Sham, Phys. Rev. 140, A1133 (1965).

[49] P. E. Blöchl, Phys. Rev. B 50, 17953 (1994).

[50] G. Kresse and D. Joubert, Phys. Rev. B 59, 1758 (1999).

[51] S. H. Vosko, L. Wilk, and M. Nusair, Can. J. Phys. 58, 1200 (1980).

[52] Z.-Y. Zhang, C. Miao, and W. Guo, Nanoscale 5, 11902 (2013).

[53] L. Algharagholy, T. Pope, Q. Al-Galiby, H. Sadeghi, S. W. D. Bailey, and C. J. Lambert, J. Mater. Chem. C 3, 10273 (2015).

[54] X. Zhang, G. Yu, X. Huang, and W. Chen, Phys. Chem. Chem. Phys. 19, 17834 (2017).

[55] L. Kong, J. R. Chelikowsky, J. B. Neaton, and S. G. Louie, Phys. Rev. B 76, 235422 (2007). 